\title{
The ExoMolOP database: Cross sections and $k$-tables for molecules of interest in high-temperature exoplanet atmospheres ${ }^{\star}$
} \author{
Paul Mollière ${ }^{3}$, Ahmed F. Al-Refaie ${ }^{2}$, Mark W. Phillips ${ }^{4}$, and Jonathan Tennyson ${ }^{2}$ \\ 1 SRON Netherlands Institute for Space Research, Sorbonnelaan 2, 3584, CA, Utrecht, The Netherlands \\ e-mail: k.l.chubb@sron.nl \\ 2 Department of Physics and Astronomy, University College London, London WC1E 6BT, UK \\ e-mail: katy.chubb.14@ucl.ac.uk \\ 3 Max-Planck-Institut für Astronomie, Königstuhl 17, 69117 Heidelberg, Germany \\ 4 Astrophysics Group, School of Physics, University of Exeter, Stocker Road, Exeter EX4 4QL, UK
}

Katy L. Chubb ${ }^{1,2}$, Marco Rocchetto ${ }^{2}$, Sergei N. Yurchenko ${ }^{2}$, Michiel Min ${ }^{1}$, Ingo Waldmann ${ }^{2}$, Joanna K. Barstow ${ }^{2}$,

Received 5 May 2020 / Accepted 28 August 2020

\begin{abstract}
Here we present a publicly available database of opacities for molecules of astrophysical interest named ExoMolOP that has been compiled for over 80 species, and is based on the latest line list data from the ExoMol, HITEMP, and MoLLIST databases. These data are generally suitable for characterising high-temperature exoplanet or cool stellar and substellar atmospheres, and have been computed at a variety of pressures and temperatures, with a few molecules included at room temperature only from the HITRAN database. The data are formatted in different ways for four different exoplanet atmosphere retrieval codes; ARCiS, TauREx, NEMESIS, and petitRADTRANS, and include both cross sections (at $\left.R=\frac{\lambda}{\Lambda 1}=15000\right)$ and $k$-tables (at $R=\frac{\lambda}{\Lambda \lambda}=1000$ ) for the $0.3-50 \mu \mathrm{m}$ wavelength region. Opacity files can be downloaded and used directly for these codes. Atomic data for alkali metals $\mathrm{Na}$ and $\mathrm{K}$ are also included, using data from the NIST database and the latest line shapes for the resonance lines. Broadening parameters have been taken from the literature where available, or have been estimated from the parameters of a known molecule with similar molecular properties where no broadening data are available.
\end{abstract}

Key words. molecular data - opacity - radiative transfer - planets and satellites: atmospheres planets and satellites: gaseous planets - infrared: planetary systems

\section{Introduction}

There is now a large amount of molecular line list data available for characterising hot exoplanet or cool stellar/substellar atmospheres, largely due to databases such as ExoMol (Tennyson \& Yurchenko 2012; Tennyson et al. 2016), HITEMP (Rothman et al. 2010; Hargreaves et al. 2019), MoLLIST (Bernath 2020), and TheoReTs (Rey et al. 2016). Line lists are independent of temperature and pressure, and so provide the most efficient way of storing the information required for characterising high-temperature astrophysical atmospheres. In order to convert this line list data into opacities (cross sections or $k$-tables), software such as ExoCross (Yurchenko et al. 2018a) is required to convert a pressure and temperature independent line list to cross-section data at a particular pressure and temperature. If a large number of pressures and temperatures are required for a large number of molecules, this can be a computationally demanding task. The present opacity database was formed in order to help reduce the computational effort of the community and to allow quick download and use of the data for many molecules designed specifically for use in atmospheric retrieval codes. The data are stored in formats which are exactly compatible for use with four different retrieval codes; ARCiS (Min et al. 2020), TauREx (Waldmann et al 2015a,b; Al-Refaie et al. 2019), NEMESIS (Irwin et al. 2008), and petitRADTRANS (Mollière et al. 2019). Retrieval codes such as these have their own processes for the computation of opacities, but have previously been limited to a subsection of

\footnotetext{
$\star$ The data are available from www. exomol. com.
}

the molecules for which data are available. The data format required to input the opacities into each retrieval code is detailed in Sect. 4, with the intention that the data files are sufficiently easy to manipulate and reformat for use in any general retrieval code. There are tools available online, such as the exo-k library ${ }^{1}$ (Leconte et al., in prep.), which enable conversion between different formats, some of which are those used in this work. Many other works have computed opacities for use in radiativetransfer retrieval and atmospheric modelling codes; see, for example, Showman et al. (2009), Freedman et al. (2008, 2014), Lee et al. (2019), Amundsen et al. (2014), Kempton et al. (2017), Grimm \& Heng (2015), Malik et al. (2019), Kitzmann et al. (2020), Allard et al. (2012), Sharp \& Burrows (2007), Line et al. (2013), Gandhi \& Madhusudhan (2017), Phillips et al. (2020), Jørgensen (1998), Kurucz \& Bell (1995).

The database of cross sections and $k$-tables presented in this work were used in a recent study by Chubb et al. (2020a), who re-examined the transmission spectra of "Hot Jupiter" exoplanet WASP-43b. These authors found that AlO, which had not previously been considered in similar analyses of the transmission spectra (Kreidberg et al. 2014; Stevenson et al. 2017; Fisher \& Heng 2018; Tsiaras et al. 2018; Weaver et al. 2019; Irwin et al. 2020), was the molecule that fitted the data to the highest level of confidence out of all molecules for which high-temperature opacity data currently exist in the infra-red region covered by the HST WFC3 instrument

\footnotetext{
http://perso. astrophy.u-bordeaux.fr/ jleconte/exo_ $\mathrm{k}$-doc/index.html
} 
(Bean 2013). Other molecules with absorption features in this 1.1-1.7 $\mu \mathrm{m}$ region include $\mathrm{C}_{2} \mathrm{H}_{2}, \mathrm{HCN}, \mathrm{FeH}, \mathrm{NH}_{3}, \mathrm{ScH}$, VO, and TiH (Tennyson \& Yurchenko 2018). Opacities from this database have also been used in works related to the investigation of ions in the thermospheres of planets (Bourgalais et al. 2020), the modelling of brown dwarf atmospheres (Lee et al. 2020), and other works related to exoplanet atmospheres (Taylor et al., in prep; Min et al. 2020).

This paper is structured as follows. Section 2 gives an introduction to the line lists used in the present database and their data format. Section 3 discusses the computation of cross sections and $k$-tables from these line lists, including details of line broadening parameters used for each species in Sect. 3.2. Section 4 gives an overview of the four retrieval codes for which these opacities are formatted, and the data format specifications of each opacity file. The line list sources used for each species and their properties are detailed in Sect. 5, with comments in Sect. 5.1, and isotopologues in Sect. 5.2. The wavelength coverage of the database is briefly discussed in Sect. 6. Section 7 summarises the ExoMolOP database, including access and upkeep. The opacity requirements for high-resolution studies are discussed in Sect. 8. We give our conclusions in Sect. 9.

\section{Line lists}

\subsection{Sources}

HITRAN (Gordon et al. 2017) is a database of largely experimental data taken at room temperature. For this reason, although it is often very accurate - more accurate than theoretically calculated data - it is not considered complete (many of the weaker lines in particular are missing); it is only designed for temperatures in the region of $296 \mathrm{~K}$ although in practice HITRAN should work satisfactorily for temperatures below this. The GEISA database (Jacquinet-Husson et al. 2016) has similar properties to HITRAN. In order to characterise high-temperature atmospheres, such as those of exoplanets and stars, theoretical calculations need to be used in order to compute energy levels up to high energies, along with the Einstein-A coefficients between them (giving the probability of a transition between two states). Large projects and associated databases which contain line list data appropriate up to much higher temperatures (at least $1000 \mathrm{~K}$ ) have therefore been set up to this effect. These include ExoMol (Tennyson \& Yurchenko 2012; Tennyson et al. 2016), HITEMP (Rothman et al. 2010; Hargreaves et al. 2019), MoLLIST (Bernath 2020), along with TheoReTS (Rey et al. 2016), SPECTRA (Mikhailenko \& Babikov 2005), MeCaSDA, and ECaSDa (Ba et al. 2013). It should be noted that a small number of molecules in the HITRAN database $(\mathrm{HF}, \mathrm{HCl}, \mathrm{HBr}$, $\mathrm{HI}, \mathrm{H}_{2}$ ) are considered applicable up to high temperatures of around $4000-5000 \mathrm{~K}$ ( $\mathrm{Li}$ et al. 2013). The data for this work were sourced mainly though the ExoMol database, with additions from HITEMP and MoLLIST (and HITRAN for the above-mentioned molecules for which data are appropriate up to $5000 \mathrm{~K}$ ) where data are not currently available from ExoMol, or where they are more complete and therefore recommended for use from a different source. Atomic data are taken from the NIST database (Kramida et al. 2013), with original doublet data measured by Juncar et al. (1981) and Falke et al. (2006).

\subsection{Data format}

As stated above, the main data sources for this work are ExoMol, HITEMP/HITRAN, and MoLLIST. The data format of ExoMol line lists is explained in detail by Tennyson et al. (2013, 2016), with a 2020 update in Tennyson et al. (2020). A summary of the format is given below:

- A ".states" file, giving a unique set of energy levels for each molecule, along with a full set of quantum numbers;

- a ".trans" file, giving the transition probabilities between allowed energy states, in the form of Einstein-A coefficients;

- (for some molecules) a ".broad" file, describing the broadening parameters (typically for self, air, $\mathrm{H}_{2}$, or He broadening) for the transitions, as a function of rotational quantum number $J$;

- a ".pf" file, giving the temperature-dependent partition function.

The HITRAN and HITEMP data format, on the other hand, consists of one transition file per molecule with each line in the file representing one transition (i.e. one line of the spectra). All the quantum numbers for the upper- and lower-energy states are contained on this same line, along with any broadening parameters. This format works for HITRAN and HITEMP databases due to their much smaller size in comparison to ExoMol line lists. The number of lines in an ExoMol line list is typically many billion for larger polyatomic molecules, and therefore it not feasible to store such a large amount of data in HITRAN/HITEMP format.

\section{Computing opacities}

\subsection{Cross sections}

In order to compute cross sections from these line lists, we made use of ExoCross (Yurchenko et al. 2018a), which is a Fortran code for generating spectra (absorption and emission) and thermodynamic properties from molecular line lists, and accepts several formats including those of ExoMol and HITRAN/HITEMP. It produces cross sections at a specified resolution (or number of points), and broadening parameters can be included, with a variety of line broadening schemes available (see Sect. 3.2). The MoLLIST data used in this work were converted to ExoMol format by Wang et al. (2020). ExoCross is also capable of working with the super-lines method of Rey et al. (2016); see Sect. 3.2.4.

The partition functions for those species with data from HITRAN or HITEMP were mainly computed using TIPS (Total Internal Partition Sums) from Gamache et al. (2017), while for data which taken from the online ExoMol portal ${ }^{2}$, the partition function provided there was used.

Rocchetto (2017) found that while a sampling resolving power of $R=\frac{\lambda}{\Delta \lambda}=10000$ was sufficient to retrieve WFC3/HST spectra, a resolving power of at least 15000 is needed to model JWST spectra. We therefore used $R=15000$ for the crosssection data presented in this work. This resolution is still insufficient for high- resolution Doppler shift studies; for this see the recent work by Gandhi et al. (2020).

The cross sections for each species are computed at the grid of 27 temperature and 22 pressures as given by Table 1, giving a total of 594 temperature-pressure combinations for each molecule. The minimum and maximum wavelength values at which cross sections are computed is between 0.3 and $50 \mu \mathrm{m}$ $\left(200-33333 \mathrm{~cm}^{-1}\right)$, although not all molecules have this wide coverage (see Tables 7-14 in Sect. 5). Figures 1 and 2 provide an illustration of $\mathrm{H}_{2} \mathrm{O}$ cross sections using the POKAZATEL line list (Polyansky et al. 2018) computed at a variety of pressures for $T=1000 \mathrm{~K}$ and a variety of temperatures for $P=0.1 \mathrm{bar}$,

\footnotetext{
WWW . exomol.com
} 
Table 1. Temperatures and pressures at which the cross sections and $k$-tables presented in this work are calculated.

\begin{tabular}{lcccccc}
\hline \hline $\begin{array}{l}\text { Temperatures } \\
(\mathrm{K})\end{array}$ & 100 & 200 & 300 & 400 & 500 & 600 \\
& 700 & 800 & 900 & 1000 & 1100 & 1200 \\
& 1300 & 1400 & 1500 & 1600 & 1700 & 1800 \\
& 1900 & 2000 & 2200 & 2400 & 2600 & 2800 \\
& 3000 & 3200 & 3400 & & \\
\hline Pressures & $1 \times 10^{-5}$ & $2.1544 \times 10^{-5}$ & $4.6416 \times 10^{-5}$ \\
(bar) & $1 \times 10^{-4}$ & $2.1544 \times 10^{-4}$ & $4.6416 \times 10^{-4}$ \\
& $1 \times 10^{-3}$ & $2.1544 \times 10^{-3}$ & $4.6416 \times 10^{-3}$ \\
& $1 \times 10^{-2}$ & $2.1544 \times 10^{-2}$ & $4.6416 \times 10^{-2}$ \\
& $1 \times 10^{-1}$ & $2.1544 \times 10^{-1}$ & $4.6416 \times 10^{-1}$ \\
& 1 & 2.1544 & 4.6416 \\
& 10 & 21.544 & 46.416 \\
& 100 & & & & \\
& & & &
\end{tabular}

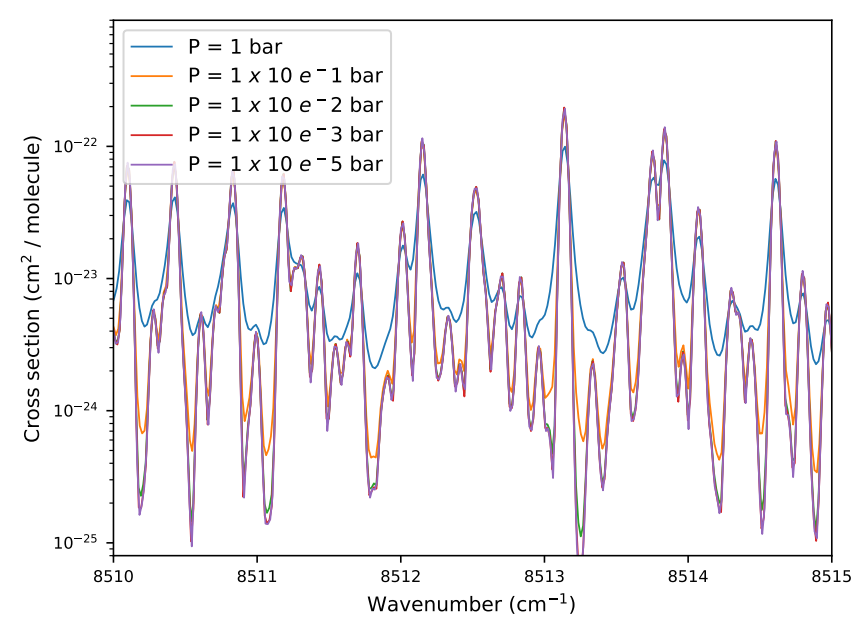

Fig. 1. $\mathrm{H}_{2} \mathrm{O}$ broadened by $\mathrm{H}_{2}$ and $\mathrm{He}$ (using the parameters of Table 2) at a variety of pressures for $T=1000 \mathrm{~K}$.

respectively. The broadening parameters of Table 2 are used, assuming a solar $\mathrm{H}_{2}$ :He ratio (see Sect. 3.2).

Different errors resulting from various aspects of computed cross sections have been explored by Hedges \& Madhusudhan (2016), Rocchetto (2017), Gharib-Nezhad \& Line (2019) and Barstow et al. (2020). Differences largely arise from either the choice of line list for a particular species, or from the different treatments of the broadening of the lines, including linewing cut-offs. The differences resulting from broadening type (i.e. self- compared to $\mathrm{H}_{2} / \mathrm{He}$ broadening) are thought to be significant for some species, such as $\mathrm{H}_{2} \mathrm{O}$, but not so much for others like $\mathrm{CH}_{4}, \mathrm{CO}_{2}$, or $\mathrm{CO}$ (Gharib-Nezhad \& Line 2019).

It is well-known that a higher sampling of spectral lines leads to improvements in the accuracy of the opacity. Every line being very well sampled is equivalent to line-by-line integration. However, sampling cross sections at a lower resolution is far more computationally feasible for atmospheric retrievals. Retrieval results using cross sections have been found to be generally good when the sampling resolution is around two orders of magnitude higher than the resolution of the observed spectrum (Rocchetto 2017). For this work, high-resolution cross sections were first computed for each pressure-temperature grid point (as determined by Table 1) and then sampled to a lower resolution

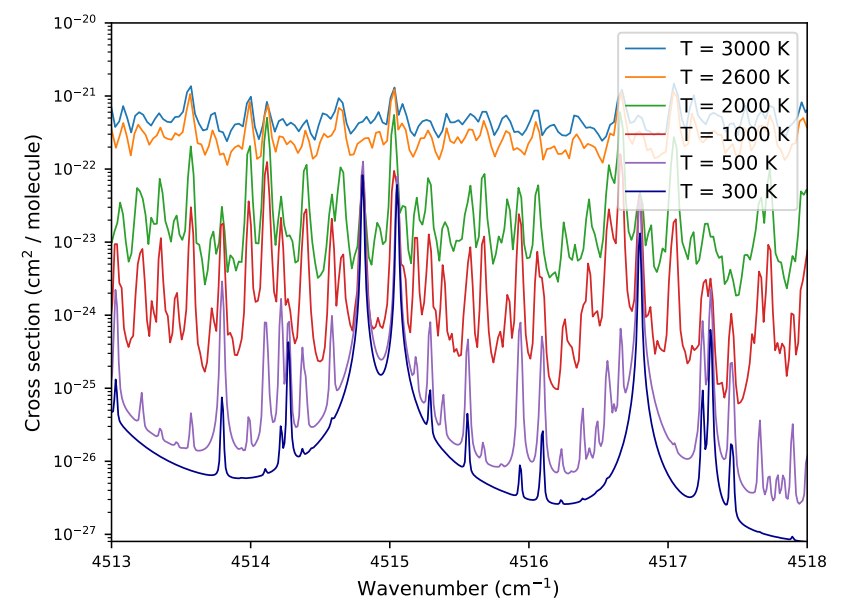

Fig. 2. $\mathrm{H}_{2} \mathrm{O}$ broadened by $\mathrm{H}_{2}$ and $\mathrm{He}$ (using the parameters of Table 2) at a variety of temperatures for $P=0.1$ bar.

cross section or $k$-tables (see Sect. 3.3). The line broadening parameters used for these high-resolution cross sections are discussed in Sect. 3.2. For some molecules, such as those with many millions or billions of lines, the super-lines method was used (see Sect. 3.2.4), which has been found to vastly improve efficiency of calculations, and yields a very small error transmission provided a sufficiently fine grid is used (Yurchenko et al. 2017a).

See Sect. 3.2 for a summary of the method used to try and ensure an adequate number of sampling points for the Voigtbroadened high-resolution cross sections, depending on wavelength region, pressure, and temperature.

\subsection{Line broadening}

A line list can be used to compute a simple stick spectra; a temperature-dependent list of line positions and line intensities. However, a real spectrum will always have some broadening of these spectral lines due to various processes. The dominant type of line broadening in an exoplanet or stellar atmosphere are Doppler (thermal) and pressure broadening. Doppler broadening, which is temperature-dependent, arises because of the thermal velocities of individual molecules, and is represented by a Gaussian line profile (see, e.g. Yurchenko et al. 2018a):

$f_{\tilde{v}_{f i}, \alpha_{D}}^{D}(\tilde{v})=\sqrt{\frac{\ln 2}{\pi}} \frac{1}{\alpha_{D}} \exp \left(-\frac{\left(\tilde{v}-\tilde{v}_{f i}\right)^{2} \ln 2}{\alpha_{D}^{2}}\right)$,

where $\tilde{v}_{f i}$ is the position of the line centre and $\alpha_{D}$ is the Doppler half-width at half-maximum (HWHM), as given by:

$\alpha_{D}=\sqrt{\frac{2 k_{B} T \ln 2}{m}} \frac{\tilde{v}_{f i}}{c}$

for a molecule of mass $m$, at temperature $T$, and with $k_{B}$ and $c$ representing the Boltzmann constant and speed of light, respectively.

Pressure broadening, which is dependent on the perturbing species (commonly $\mathrm{H}, \mathrm{He}$, air or self) as well as the pressure, leads to a Lorentzian profile, as given by:

$f_{\tilde{v}_{f i}, \gamma_{L}}^{L}(\tilde{v})=\frac{1}{\pi} \frac{\gamma_{L}}{\left(\tilde{v}-\tilde{v}_{f i}\right)^{2}+\gamma_{L}^{2}}$,

where the Lorentzian line width (HWHM) is given by:

$\gamma_{L}=\gamma_{L}^{0}\left(\frac{T_{0}}{T}\right)^{n_{L}} \frac{P}{P_{0}}$. 
Table 2. Molecular properties for the species with computed opacities presented in this work for which $\mathrm{H}_{2}$ and He broadening parameters are available in the literature, the majority of which were collated by Barton et al. (2017a).

\begin{tabular}{lccccccccc}
\hline \hline Species & $\gamma_{\mathrm{H}_{2}}$ & $n_{\mathrm{H}_{2}}$ & $\gamma_{\mathrm{He}}$ & $n_{\mathrm{He}}$ & $\begin{array}{c}\mathrm{DM} \\
(\mathrm{d})\end{array}$ & $\begin{array}{c}\mathrm{MM} \\
\left(\mathrm{g} \mathrm{mol}^{-1}\right)\end{array}$ & AN & Structure & Dipole Ref \\
\hline $\mathrm{H}_{2}$ & 0.01 & 0.13 & 0.01 & 0.13 & 0 & 2.0 & 2 & Nonpolar & Johnson-III (2019) \\
$\mathrm{CH}_{4}$ & 0.06 & 0.60 & 0.03 & 0.30 & 0 & 16.0 & 10 & Nonpolar & \\
$\mathrm{C}_{2} \mathrm{H}_{2}$ & 0.09 & 0.59 & 0.04 & 0.44 & 0 & 26.0 & 14 & Linear & Johnson-III (2019) \\
$\mathrm{CO}$ & 0.07 & 0.65 & 0.05 & 0.60 & 0.1 & 28.0 & 14 & Diatomic & \\
$\mathrm{PH}_{3}$ & 0.10 & 0.75 & 0.05 & 0.30 & 0.6 & 34.0 & 18 & Non-linear & Dijkerman \& Ruitenberg (1969) \\
$\mathrm{OCS}_{\mathrm{HCl}}$ & 0.05 & 0.75 & 0.04 & 0.75 & 0.7 & 60.1 & 30 & Linear & Johnson-III (2019) \\
$\mathrm{NH}_{3}$ & 0.03 & 0.75 & 0.01 & 0.75 & 1.1 & 36.5 & 18 & Diatomic & \\
$\mathrm{SO}_{2}$ & 0.14 & 0.50 & 0.03 & 0.50 & 1.5 & 17.0 & 10 & Non-linear & \\
$\mathrm{H}_{2} \mathrm{O}$ & 0.06 & 0.20 & 0.07 & 0.64 & 1.6 & 64.1 & 32 & Non-linear & \\
$\mathrm{HF}$ & 0.04 & 0.75 & 0.01 & 0.50 & 1.9 & 20.0 & 10 & Diatomic & Johnson-III (2019) \\
$\mathrm{H}_{2} \mathrm{CO}$ & 0.14 & 0.50 & 0.06 & 0.50 & 2.3 & 30.0 & 16 & Non-linear & Tomasevich (1970) \\
$\mathrm{HCN}$ & 0.12 & 0.50 & 0.05 & 0.50 & 3.0 & 27.0 & 14 & Linear & Ton \\
\hline
\end{tabular}

Notes. $\gamma_{x}$ and $n_{x}$ (where $x=\mathrm{H}$ or He) are the broadening parameters for hydrogen and helium $\left(\gamma_{0}\right.$ and $n_{L}$ in Eq. (4)). DM is the dipole moment, $\mathrm{MM}$ is the molar mass, and AN is the atomic number. The citations for the $\mathrm{H}_{2} / \mathrm{He}$ broadening parameters are given below the table for each molecule. An average value for all values of $J$ is taken for each set of broadening parameters. The reference temperature of $T_{0}=296 \mathrm{~K}$ is used in all cases.

References. $\mathrm{H}_{2}$ : Wcisło et al. (2016). $\mathrm{CH}_{4}$ : Varanasi \& Chudamani (1990), Pine (1992), Fox et al. (1998), Varanasi \& Tejwani (1972), Varanasi \& Chudamani (1989), Grigoriev et al. (2001), Gabard et al. (2004), Manne et al. (2017), Gharib-Nezhad et al. (2019) $\mathrm{C}_{2} \mathrm{H}_{2}, \mathrm{OCS} \mathrm{NH}_{3}$, $\mathrm{SO}_{2}$, HF: Wilzewski et al. (2016) HCl: Wilzewski et al. (2016), Li et al. (2018) CO: Faure et al. (2013), Li et al. (2015), Mulvihill et al. (2018), Mantz et al. (2005), Predoi-Cross et al. (2016), Sinclair et al. (1998) $\mathrm{PH}_{3}$ : Kleiner et al. (2003), Levy et al. (1993), Sergent-Rozey et al. (1988), Salem et al. (2004, 2005) $\mathrm{H}_{2}$ O: Voronin et al. (2010, 2012), Barton et al. (2017b), Solodov \& Starikov (2008, 2009), Petrova et al. (2013, 2012, 2016), Gamache et al. (2019) $\mathrm{H}_{2}$ CO: Nerf (1975) HCN: Mehrotra et al. (1985), Cohen \& Wilson (1973), Charron et al. (1980).

Here, $T_{0}$ and $P_{0}$ are the reference temperature and pressure, respectively. $\gamma_{L}^{0}$ and $n_{L}$ are the reference HWHM and temperature exponent, respectively. The latter two terms are known as pressure broadening parameters, and are dependent on the molecular species being broadened and the species causing the broadening. There is also some dependence on the rotational angular momentum quantum numbers, $J$, of the states involved in a particular transition.

A convolution of the two profiles given in Eqs. (1) and (3) gives a Voigt profile, which is commonly used to represent line broadening in exoplanet atmospheres:

$f_{\tilde{v}_{f i}, \alpha_{D}, \gamma_{L}}^{V}(\tilde{v})=\frac{\gamma \sqrt{\ln 2}}{\pi^{\frac{3}{2}} \alpha_{D}} \int_{-\infty}^{\infty} \frac{e^{-y^{2}} \mathrm{~d} y}{(v-y)^{2}+\gamma^{2}}$,

where $\gamma=\sqrt{\ln 2}\left(\gamma_{L} / \alpha_{D}\right)$ and $v=\sqrt{\ln 2}\left(\tilde{v}-2 \tilde{v}_{f i}\right) / \alpha_{D}$, with terms as defined in Eqs. (1)-(4). Technically there should be a pressure-shift of the transition wavenumber, $\tilde{v}_{f i}$, included in computations of line broadening. However, there are currently large experimental uncertainties associated with the relatively small number of pressure-shift values that are currently available. There is work ongoing to improve upon these parameters (e.g. Hargreaves et al. 2019; Gamache \& Vispoel 2018), and we hope to include more accurate parameters such as these in future opacity calculations.

The efficient and accurate numerical computation of such a profile has been the subject of a number of publications (see, e.g. Hedges \& Madhusudhan 2016; Min 2017; Schreier 2017; Grimm \& Heng 2015; Yurchenko et al. 2018a; Amundsen et al. 2014). The coefficients $\gamma_{0}$ and $n_{L}$ in Eq. (4) are dependent both on the species being broadened and on the species causing the broadening (e.g. $\mathrm{H}_{2}$ and $\mathrm{He}$ are assumed to be the main broadeners in typical Hot Jupiter atmospheres (Hedges \& Madhusudhan 2016)), along with the rotational angular momentum quantum numbers, $J$, of the states involved in a particular transition.
As mentioned in Sect. 3.1, the number of sampling points required to give well-sampled Voigt-broadened high-resolution cross sections can be estimated as a function of wavelength $v$, temperature $T$, and pressure $P$, with an average number found for a given wavelength region. The Voigt width of a particular line can be approximated using the following expression (Olivero \& Longbothum 1977; Rocchetto 2017):

$\gamma_{V} \approx 0.5346 \gamma_{L}+\sqrt{0.2166 \gamma_{L}^{2}+\gamma_{G}^{2}}$

where $\gamma_{L}$ and $\gamma_{G}$ are given by Eqs. (2) and (4), respectively. In this work, we aim for an average of four sampling points per line for the initial set of high-resolution cross sections. This is estimated using Eq. (6), as a function of pressure, temperature, and wavelength region. We use a line-wing cut-off of $500 \gamma_{V}$, which is also calculated using Eq. (6) and is therefore dependent on pressure, temperature, and wavelength.

Where available, broadening parameters $\left(\gamma_{0}\right.$ and $n_{L}$ in Eq. (4)) are provided as part of the ExoMol database (see Yurchenko et al. 2017b; Barton et al. 2017a). However, in general, these broadening parameters are not well known (or given in the literature at all) for a large number of species, particularly for $\mathrm{He}$ and $\mathrm{H}_{2}$ as broadeners. In this work we aim to use approximate pressure broadening parameters with $\mathrm{He}$ and $\mathrm{H}_{2}$ as the broadening species (as they are thought to be the main constituents of Hot Jupiter atmospheres), at solar $\mathrm{H}_{2}: \mathrm{He}$ ratio, based on those parameters that do exist in the literature, and using molecular properties as given in Table 2 and Tables A.1-A.11 to estimate which parameters to use for those that do not. Table 2 lists those species for which broadening parameters exist in the ExoMol database (primarily based on the work of Barton et al. 2017a). These parameters are usually given as a function of rotational angular momentum quantum number, $J$, for each species. Here, we have taken an average value for all values of $J$ for a 


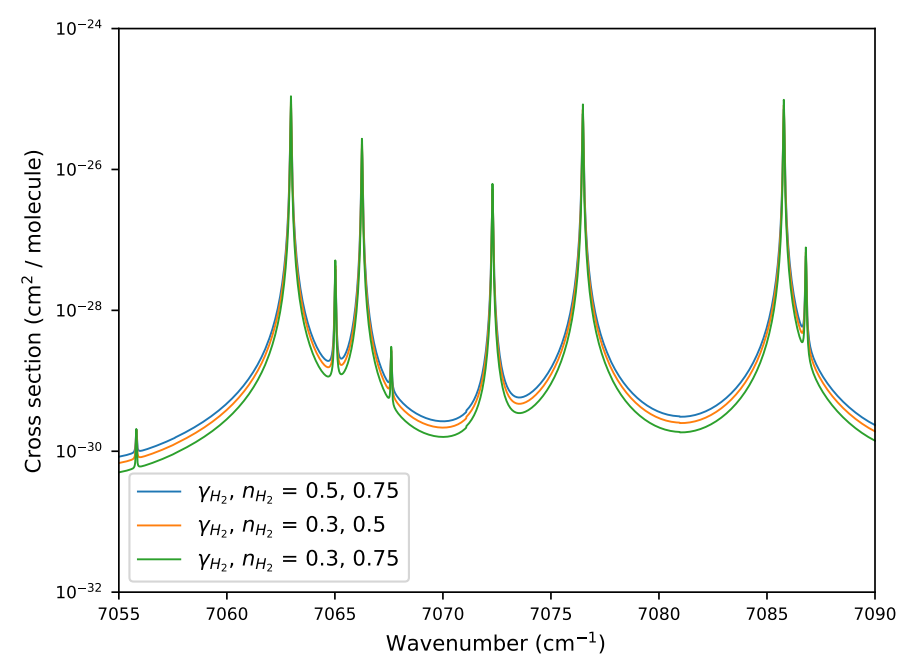

Fig. 3. $\mathrm{HCl}$ broadened by $\mathrm{H}_{2}$ using different broadening parameters. The cross sections are computed at $T=1000 \mathrm{~K}$ and $P=0.1 \mathrm{bar}$, which are typical values for a layer of atmosphere of a Hot Jupiter exoplanet being observed in this wavelength region.

given species for broadening of both $\mathrm{H}_{2}$ and He. When computing the cross sections, we then weight these parameters by assumed broadener abundances based on the solar $\mathrm{H}_{2}: \mathrm{He}$ ratio. When deciding which species is most similar from a broadening point of view to those in Table 2, we consider the following factors. First, we consider the dipole moment (DM), and quadrupole moment (QM) where $D M=0$ (Buldyreva et al. 2010a). We then also look at the general structure (e.g. linear, non-linear, diatomic), and consider molecular properties such as the centre of symmetry and interatomic distances. Of the non-polar $(D M=0)$ species in Table $2, \mathrm{CH}_{4}$ has $Q M=0, \mathrm{H}_{2}$ has a small $\mathrm{QM}$, and $\mathrm{C}_{2} \mathrm{H}_{2}$ has a relatively large QM. The other non-polar molecules are grouped accordingly, based on whether their QM is zero, low, or high. The reference for the value of the dipole moment in these tables is given where applicable. For those with no obvious value in the literature, the dipole moment was computed using MOLPRO (Werner et al. 2012), with a cc-pVTZ basis set at CASSCF level of theory (Olsen 2011). Where multiple values of dipole moment are given in the literature (depending on the level of theory used to calculate it), we used an average value. It is stressed that these values are only given as a guide towards determining which species in Table 2 is most similar to those in Tables A.1-A.11, and should not be taken as exact. For those diatomic species in Table 2 where line-by-line broadening parameters are provided (e.g. by Li et al. 2015; Wilzewski et al. 2016; Gordon et al. 2017), we use these values instead of the average parameters when computing their opacities.

Figure 3 illustrates the effect of using different broadening parameters for $\mathrm{HCl}$ when computing the cross sections for a pressure and temperature typical of a Hot Jupiter exoplanet being observed in the given wavelength region. The broadening is considered to be from $\mathrm{H}_{2}$ only in this example.

Studies such as those by Hedges \& Madhusudhan (2016), Rocchetto (2017), Baudino et al. (2017), Gharib-Nezhad \& Line (2019), and Barstow et al. (2020) have highlighted the differences in forward models and retrievals caused by the choice of molecular broadening parameters. It is important to note that although there is a known difference between different parameters, there is still much work required in order to determine the "true" parameters, or even a good approximation, for many species. The requirement for enhanced line-broadening parameters was recently identified by Fortney et al. (2019) in their study of the need for laboratory data requirements for studies of exoplanetary atmospheres. This is the focus of various works such as Stolarczyk et al. (2020), Hartmann et al. (2018), who are improving upon the current knowledge of molecular broadening. Some detailed accounts of line-broadening theory can be found in, for example, Buldyreva et al. (2010a,b) and Wcisło et al. (2016). The latter demonstrates that $\mathrm{H}_{2}$ has "exceptionally pronounced non-Voigt line-shape effects". We do not take these more precise effects into account in this work, but they could be considered in future work. Other intricacies have been neglected here. We note, for example, that broadening parameters are not only dependent on $J$, but also on the lower state symmetry (see, e.g. Gharib-Nezhad et al. 2019). However, the main focus of this work is towards useable opacities for low-resolution atmospheric retrieval studies. There are many other limiting factors when it comes to detecting molecules and making accurate determinations of their abundances. The accurate treatment of pressure effects is beyond the scope of the current work, but is planned to be significantly enhanced in future releases of the ExoMol and therefore ExoMolOP database.

\subsection{1. $\mathrm{H}_{2} \mathrm{~S}$ : A broadening case study}

We can compare the molecular properties of $\mathrm{H}_{2} \mathrm{~S}$ to those of the species listed in Table 2 which have some broadening parameters available in the literature. The dipole moment of $\mathrm{H}_{2} \mathrm{~S}$ is closest to $\mathrm{HCl}$. However, the intermolecular distances and potential energy surface of $\mathrm{H}_{2} \mathrm{~S}$ are more similar to OCS than $\mathrm{HCl}$ (Johnson-III 2019), and so it is not clear which broadening parameters should be used. Here, we compute opacities using the two different sets of broadening parameters $\left(\gamma_{\mathrm{H}_{2}}, n_{\mathrm{H}_{2}}, \gamma_{\mathrm{He}}\right.$, $n_{\mathrm{He}}=0.03,0.75,0.01,0.75$ and $0.05,0.75,0.04,0.75$, for $\mathrm{HCl}$ and OCS, respectively) in order to illustrate the effects of using different broadening parameters. For $\mathrm{HCl}$, the average values of the broadening parameters are used from Wilzewski et al. (2016), with the exception of $n_{\mathrm{H}_{2}}$. This latter parameter was originally sourced by Wilzewski et al. (2016) from the work of Houdeau et al. (1980), which presents negative temperature dependence exponents. As noted by Wilzewski et al. (2016), negative exponents are not impossible but they are unexpected, particularly for a simple diatomic like $\mathrm{HCl}$. Negative exponents for $\mathrm{HCl}-\mathrm{N}_{2}$ were also previously published by Houdeau et al. (1980), which were subsequently found to be positive by Pine \& Looney (1987). This gives further cause to be cautious about using the negative values. We therefore instead use the suggested default value of $n_{\mathrm{H}_{2}}=0.75$ for $\mathrm{HCl}$. There are some values for $\gamma_{\mathrm{He}}$ (Waschull et al. 1994) and $\gamma_{\mathrm{H}_{2}}$ (Kissel et al. 2002; Starikov \& Protasevich 2006) for the $v_{2}$ vibrational band of $\mathrm{H}_{2} \mathrm{~S}$. These range from 0.04-0.07 for $\gamma_{\mathrm{H}_{2}}$ and 0.04-0.1 for $\gamma_{\mathrm{He}}$, which give further agreement with the values of OCS over those of $\mathrm{HCl}$.

Figure 4 illustrates the difference in computed cross sections of $\mathrm{H}_{2} \mathrm{~S}$ between using the broadening parameters of Table 2 for $\mathrm{OCS}$ and $\mathrm{HCl}$. A couple of different pressure-temperature combinations are used for comparison. It can be seen that the difference is more pronounced for lower temperatures and pressures, as was found in previous studies (Hedges \& Madhusudhan 2016; Rocchetto 2017). Figure 5 illustrates forward models of a hypothetical atmosphere (computed using TauREx, Waldmann et al. 2015a,b), comparing opacities computed using these two different sets of broadening parameters. The model atmosphere is computed at $1000 \mathrm{~K}$ and contains $\mathrm{H}_{2} \mathrm{~S}$ only. The differences can be seen to be small, but more pronounced at higher wavelengths. For the pressures and temperatures typical of a Hot Jupiter exoplanet 

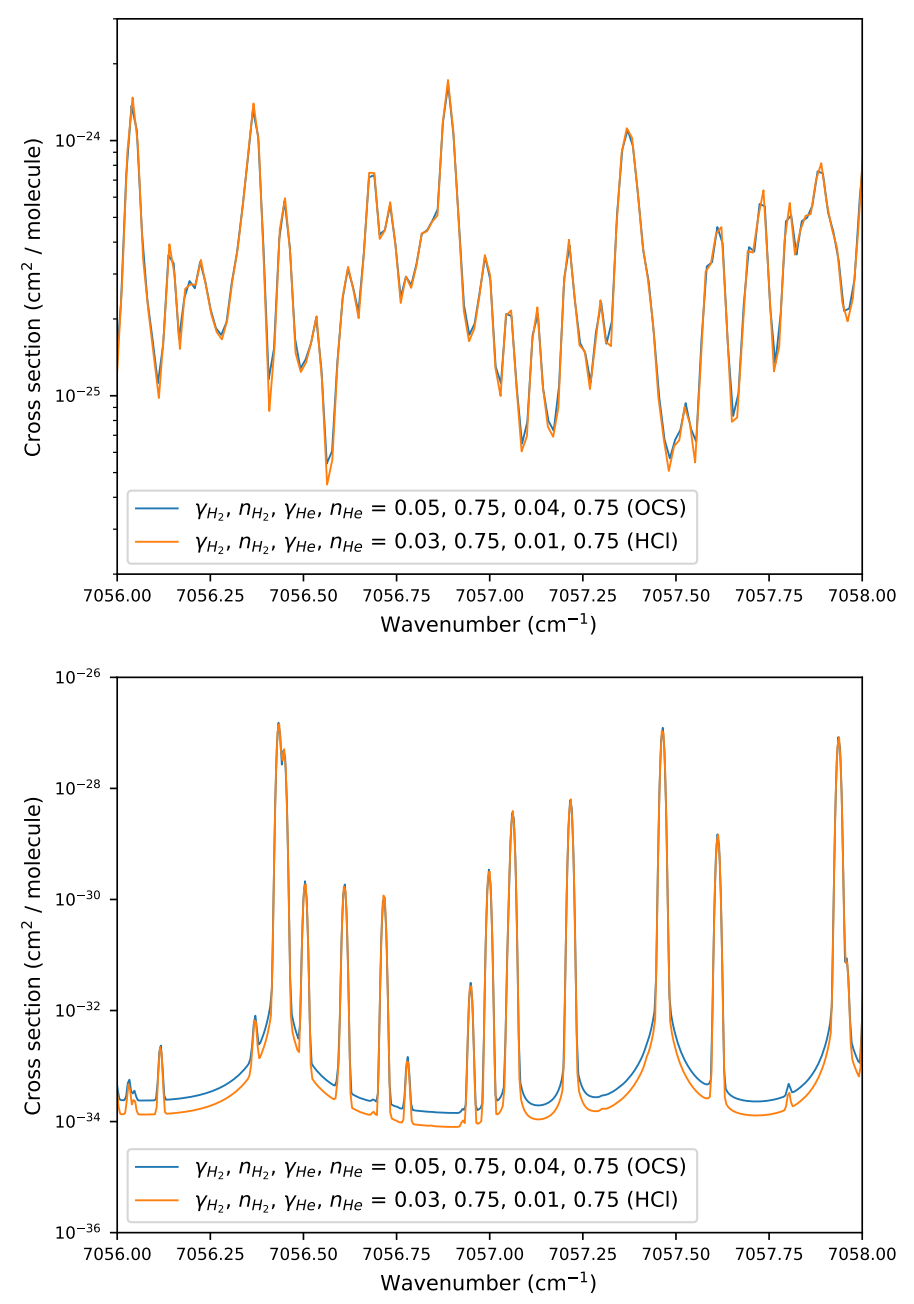

Fig. 4. $\mathrm{H}_{2} \mathrm{~S}$ broadened by $\mathrm{H}_{2}$ and $\mathrm{He}$ using different broadening parameters based on values for $\mathrm{HCl}$ and OCS. The cross sections in the top panel are computed at $T=1000 \mathrm{~K}$ and $(P=0.1$ bar), which are typical values for a layer of atmosphere of a Hot Jupiter exoplanet observed in this wavelength region. The cross sections in the bottom panel are computed at $T=100 \mathrm{~K}$ and $P=1 \times 10^{-} 5$ bar.

observed in the near-infrared, we do not expect the difference in broadening parameters to have a significant effect. Other uncertainties caused by for example incomplete line lists or line-wing cut-offs have been demonstrated to have a larger effect on the atmospheric spectrum by studies such as Rocchetto (2017) for example. However, the differences in opacities caused by the use of different broadening parameters should always be taken into consideration when interpreting and discussing results.

\subsubsection{Comparison of broadening values used here and in other studies}

Broadening parameters can be found in the literature for a small number of other species, which can then be compared to those we are using in this work. A couple of examples are given here.

The value of $\gamma_{H_{2}}$ for $\mathrm{C}_{2} \mathrm{H}_{4}$ from Brannon \& Varanasi (1992) is $\sim 0.12 \mathrm{~cm}^{-1} \mathrm{~atm}^{-1}$. We assume a value of $\gamma_{H_{2}}=0.09 \mathrm{~cm}^{-1}$ atm $^{-1}$ based on the broadening parameters of $\mathrm{C}_{2} \mathrm{H}_{2}$ from Table 2 .

The values of $\gamma_{\mathrm{H}_{2}}$ and $\gamma_{\mathrm{He}}$ for $\mathrm{CH}_{3} \mathrm{~F}$ from Lerot et al. (2006) and Grigoriev et al. (1997) are $\sim 0.14 \mathrm{~cm}^{-1} \mathrm{~atm}^{-1}$ and $\sim 0.12 \mathrm{~cm}^{-1}$ $\mathrm{atm}^{-1}$, respectively. We assume values of $\gamma_{\mathrm{H}_{2}}=0.14 \mathrm{~cm}^{-1} \mathrm{~atm}^{-1}$ and $\gamma_{\mathrm{He}}=0.06 \mathrm{~cm}^{-1} \mathrm{~atm}^{-1}$ based on the broadening parameters of
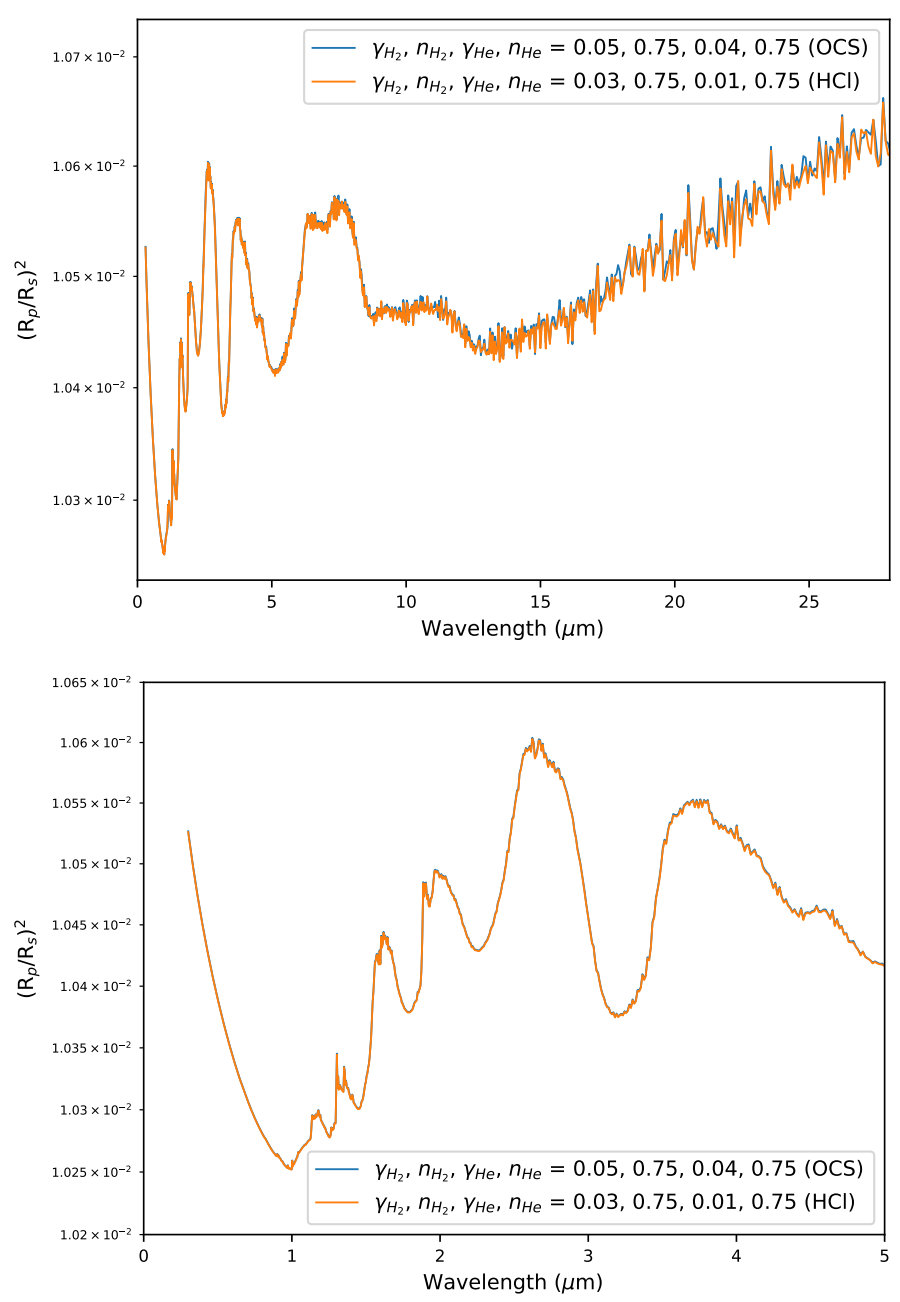

Fig. 5. Hypothetical model atmosphere of an exoplanet composed of $\mathrm{H} 2 \mathrm{~S}$ only. $\mathrm{H}_{2} \mathrm{~S}$ is broadened by $\mathrm{H}_{2}$ and $\mathrm{He}$ using different broadening parameters in the two cases shown in each panel based on values for OCS and $\mathrm{HCl}$. The model atmosphere is computed at $T=1000 \mathrm{~K}$ across pressures ranging from $1 \times 10^{-5}$ to $1 \times 10^{6}$ bar. The two panels illustrate different regions of the spectrum.

$\mathrm{H}_{2} \mathrm{CO}$ from Table 2. Figure 6 illustrates the effects on cross sections computed using these different broadening parameters for $\mathrm{C}_{2} \mathrm{H}_{4}$ (top panel) and $\mathrm{CH}_{3} \mathrm{~F}$ (bottom panel). We note that the temperature exponents are not given in the literature for these species, and so we do not have a full set of broadening parameters to add to Table 2 .

Hedges \& Madhusudhan (2016) use the following metric to quantify the effect of using different broadening parameters, which we adopt here.

$\delta=\operatorname{median}\left(\frac{\sigma-\sigma_{0}}{\sigma_{0}}\right) \times 100$,

where $\delta$ is therefore the median percent change in cross section (computed at a given pressure $P$ and temperature $T$ ), with $\sigma$ and $\sigma_{0}$ being the cross sections computed using two different sets of broadening parameters. Here we compute $\delta$ for cross sections of $\mathrm{CO}$ using the $J$-averaged broadening parameters of Table 2 compared to cross sections of CO using the $J$-dependent broadening parameters of $\mathrm{Li}$ et al. (2015). Here we are comparing the high-resolution cross sections before sampling to $k$-tables or cross sections of lower resolution. We find that $\delta$ is $0.16 \%$ for $P=0.1$ bar and $T=1000 \mathrm{~K}$, and that $\delta<0.3 \%$ for 

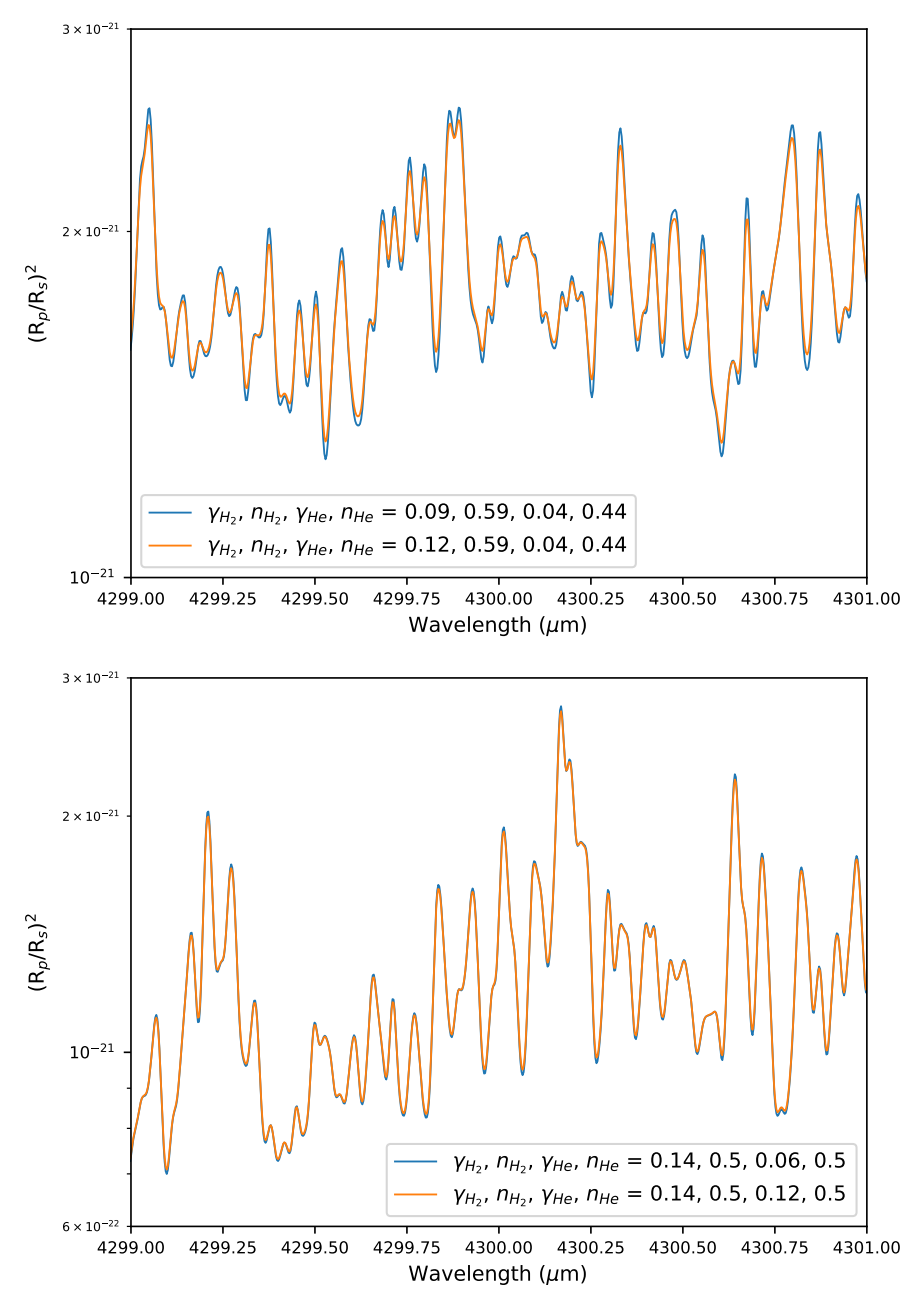

Fig. 6. $\mathrm{C}_{2} \mathrm{H}_{4}$ (top panel) and $\mathrm{CH}_{3} \mathrm{~F}$ (bottom panel) broadened by $\mathrm{H}_{2}$ and He using different broadening parameters. The cross sections are computed at $T=1000 \mathrm{~K}$ and $P=0.1 \mathrm{bar}$, which are typical values for a layer of atmosphere of a Hot Jupiter exoplanet observed in this wavelength region.

all pressure-temperature combinations considered in this work. Here, $\delta$ is highest for the lowest values of pressure and temperature. Hedges \& Madhusudhan (2016) find that the effect of using different broadening parameters is more pronounced at higher resolution. We therefore do not expect the approximations of broadening parameters used in this work to be a significant issue for low-resolution studies, particularly for high temperatures $(>1000 \mathrm{~K})$ and pressures $\left(>1 \times 10^{-3}\right.$ bar $)$. However, we are aware that theoretical and observational advances in the near future will mean that it will be beneficial to update ExoMolOP with more accurate parameters.

\subsubsection{Atomic $\mathrm{Na}$ and $\mathrm{K}$ broadening}

While the broadening parameters for molecular species are currently largely uncertain, the same is not true for the strong doublet lines in alkali metals sodium and potassium. These strong absorption features can be found in many high-temperature atmospheres; hot Jupiters (Lendl et al. 2017; Sing et al. 2014), stellar atmospheres (Takeda et al. 2012), and brown dwarfs, where they have been observed to be non-Lorentzian (Burrows et al. 2000, 2002a). Studies such as these have motivated the use of more detailed quantum chemistry calculations, which have been employed in order to accurately treat the broadening of these lines by $\mathrm{H}_{2}$ and $\mathrm{He}$ for a variety of pressures and temperatures (Peach \& Whittingham 2009; Allard et al. 2007, 2019; Burrows \& Volobuyev 2003; Peach 2017).

In this work, the pressure- and temperature-broadened profiles for the resonance doublets of $\mathrm{Na}$ and $\mathrm{K}$ are computed using Allard et al. (2016, 2019). Future updates to these opacities intend to also consider He broadening, as outlined in Peach et al. (2020). The data for all other lines are taken from NIST Kramida et al. (2013), with Voigt broadening based on parameters inferred from Allard et al. (2007), and line-wing cutoffs computed at $4500 \mathrm{~cm}^{-1}$, as recommended by Baudino et al. (2017). We do note that computing line wings out to this distance may not be wise when the profiles are not accurately known. However, preliminary tests indicate that the chosen line-wing cut-off for these non-resonance $\mathrm{K}$ lines makes no noticeable difference to model emission spectra of brown dwarf atmospheres (where the differences are more pronounced than in exoplanet atmospheres), as the non-resonant $\mathrm{K}$ lines were drowned out by other opacities. The same is assumed to be true for $\mathrm{Na}$.

\subsubsection{Super-lines}

The super-lines method (Rey et al. 2016) is a new functionality of ExoCross (Yurchenko et al. 2018a) which allows for a huge increase in computational speed and therefore efficiency. The general principle is to compute line intensities at a given temperature $T$, and then to sum together the intensities of all lines within a specified spectral bin to create a so-called super line. A Voigt broadening profile is then applied to each of these super lines. As long as each of these super lines is adequately sampled then overall opacity will be conserved. We use this method when computing opacities of larger molecules, which contain many millions to billions of lines; see Tables 9 and 13. In order to ensure error transmission is kept to a minimum, we use a grid of $R=1000000$ for the super lines (Yurchenko et al. 2017a; Tennyson et al. 2020).

\subsection{K-tables}

Once relatively high-resolution cross sections have been computed, it is reasonably simple to produce $k$-tables using what is known as the correlated $k$-distribution method; this method is well described in the literature (see, e.g. Lacis \& Oinas 1991; Pierrehumbert 2010), and is extensively used for radiative transfer calculations in the context of planetary and substellar atmospheres (see, e.g. Irwin et al. 2008; Showman et al. 2009; Freedman et al. 2008, 2014; Lee et al. 2019; Mollière et al. 2015; Amundsen et al. 2014; Sharp \& Burrows 2007; Malik et al. 2017; Drummond et al. 2016; Phillips et al. 2020). $K$-tables are generally considered faster (and more accurate for the same $R=\frac{\lambda}{\Delta \lambda}$ ) than cross sections, but they also come with their own assumptions and therefore limitations (see, e.g. Rocchetto 2017). However, these can be assumed to be negligible when compared to other unknowns. The general principle is to order spectral lines within a given spectral bin, producing a smooth cumulative distribution function to represent opacity, which can be more efficiently sampled. The number of points used for sampling within a given spectral bin is determined by a set of Gaussian quadrature points, which are assigned corresponding weights. These are often chosen so as to sample the extremes of the bin more finely so as not to miss the weakest and strongest lines (i.e. the distance between sampling points within a bin is not constant). One of the methods 
used in this work, for example, is based on the use of Gauss Legendre polynomials (see Sect. 4 for details of the opacities produced for individual retrieval codes). $K$-tables are produced using a method of opacity sampling which enables low-resolution computations while still taking strong opacity fluctuations at high resolution into account; see, for example, Min (2017). The assumption made for the $k$-distribution method that the $k$-coefficients at each Guassian quadrature point are correlated, breaks down for inhomogeneous atmospheres (Lee et al. 2019)

\section{Retrieval codes}

There are several atmospheric retrieval codes in use by the exoplanetary characterisation community that are designed to solve the radiative transfer equation by looking at the propagation of radiation through a medium. We have tailored the data computed as part of this work to be directly available in the necessary format for four such retrieval codes, with no conversion necessary. These codes are $\mathrm{ARCiS}^{3}$ (Min et al. 2020), Tau$\mathrm{REx}^{4}$ (Waldmann et al. 2015a,b; Al-Refaie et al. 2019), NEMESIS (Irwin et al. 2008), and petitRADTRANS (Mollière et al. 2019). A summary of each code is given in this section, along with the specific requirements of the data format of the input opacity files (cross sections for TauREx, and $k$-tables for the others) for each.

\subsection{TauREx}

TauREx is a modular Bayesian inverse retrieval suite optimised for speed (on CPU and GPU platforms) and ease of use. It was originally designed for retrievals of exoplanet transmission, emission, and phase-curve measurements (Al-Refaie et al. 2019; Changeat et al., in prep.), but has recently been extended to Solar System measurements (e.g. ExoMars Trace Gas Orbiter (TGO), Cann et al., in prep.). TauREx is publicly available $\mathrm{e}^{5}$ under a BSD license.

TauREx3 has recently been released by Al-Refaie et al. (2019) with vast speed improvements compared to previous versions of the code (Waldmann et al. 2015a,b). The currently available version of TauREx 3 only has support for cross-section opacities using HDF5, pickle, and Exo-Transmit (Kempton et al. 2017 ) formats. The next release (version 3.1) will include $k$-table support for both petitRADTRANS and NEMESIS formats. The cross-section data can either be streamed directly or loaded into memory. The data contained in the HDF5 file are summarised in Table 3.

\subsection{ARCiS}

ARCiS is an atmospheric modelling and Bayesian retrieval package (Min et al. 2020). Full details of the ARCiS code are presented in Min et al. (2020). The most important information can be found in Ormel \& Min (2019). The code consists of a forward modelling part based on correlated-k molecular opacities and cloud opacities using Mie and DHS (Distribution of hollow spheres; see Min et al. 2005) computations. With ARCiS one can compute cloud formation (Ormel \& Min 2019) and chemistry (Woitke et al. 2018) from physical and chemical principles. The code was benchmarked against petitCODE (Mollière et al. 2015, 2017) by Ormel \& Min (2019). For the retrieval part the MulTI-

\footnotetext{
3 https://www.exoclouds.com

4 https://taurex3-public.readthedocs.io

5 https://github.com/ucl-exoplanets/TauREx3_public
}

Table 3. Overview of the data fields contained within the HDF5 cross sections for use in the TauREx retrieval code.

\begin{tabular}{lc}
\hline \hline Field name & Description \\
\hline mol_name & Molecule name \\
key_iso_ll & ID for isotopologue and line list \\
$t$ & List of temperatures \\
$p$ & List of pressures \\
$t . u n i t s$ & Units of temperature $(\mathrm{K})$ \\
$p . u n i t s$ & Units of pressure $(\mathrm{bar})$ \\
bin_edges & Bin edges in wavenumbers $\left(\mathrm{cm}^{-1}\right)$ \\
bin_edges.units & Units of bin edges $\left(\mathrm{cm}^{-1}\right)$ \\
xsecarr & Cross-section array $(p, t$, bin_centres) \\
xsecarr.units & Cross-section units, $\mathrm{cm}^{2} / \mathrm{molecule}^{-}$ \\
mol_mass & Molecular mass in a.m.u. \\
DOI & Digital Online Identifier for line list \\
Date_ID & ID for date of creation and version \\
\hline
\end{tabular}

Table 4. Overview of the data fields contained within the fits $k$-tables for use in the ARCiS retrieval code.

\begin{tabular}{|c|c|}
\hline Field name & Description \\
\hline $\operatorname{Tmin}$ & Minimum temperature $(\mathrm{K})$ \\
\hline $\operatorname{Tmax}$ & Maximum temperature $(\mathrm{K})$ \\
\hline Pmin & Minimum pressure (bar) \\
\hline$P \max$ & Maximum pressure (bar) \\
\hline$l \_m i n$ & Minimum wavelength $(\mu \mathrm{m})$ \\
\hline$l \_\max$ & Maximum wavelength $(\mu \mathrm{m})$ \\
\hline$\overline{n T}$ & Number of temperatures \\
\hline$n P$ & Number of pressures \\
\hline nlam & Number of wavelength points \\
\hline$n g$ & Number of gauss points \\
\hline kcoeff & $k$-coefficient array $(p, t$, gauss, bin_centre $)$ \\
\hline kcoeff_units & $k$-coefficient units $\left(\mathrm{cm}^{2} /\right.$ molecule $)$ \\
\hline$t$ & List of temperatures $(\mathrm{K})$ \\
\hline$p$ & List of pressures (bar) \\
\hline bin_centres & Bin centres in wavenumber $\left(\mathrm{cm}^{-1}\right)$ space \\
\hline mol_mass & Molecular mass in a.m.u. \\
\hline$D O I$ & Digital Online Identifier for line list \\
\hline mol_name & Molecule name \\
\hline key_iso_ll & ID for isotopologue and line list \\
\hline Date_ID & ID for date of creation and version \\
\hline
\end{tabular}

Notes. Here, gauss refers to the gauss sampling points.

NEST algorithm (Feroz \& Hobson 2008; Feroz et al. 2009, 2013) is employed. Benchmarks for the retrieval have been performed in the framework of the ARIEL mission (Pascale et al. 2018), showing excellent agreement with multiple other retrieval codes. The Gauss sampling points used for the $k$-tables are based on Gauss Legendre polynomials. Opacities are in FITS format.

\subsection{NEMESIS}

NEMESIS is a planetary atmosphere radiative transfer and retrieval tool. It was originally developed for application to Solar System planets (see, e.g. Tsang et al. 2010; Fletcher et al. 2011) and has subsequently been extended and applied to exoplanets (e.g. Lee et al. 2012; Barstow et al. 2016; Krissansen-Totton et al. 2018; Irwin et al. 2020). NEMESIS can be used with either an optimal estimation (Rodgers 2000) 
Table 5. Overview of the data fields contained within the binary $k$-tables for use in the NEMESIS retrieval code.

\begin{tabular}{|c|c|}
\hline Field name & Description \\
\hline IRECO & $11+2 * \mathrm{NG}+2+\mathrm{NP}+\mathrm{NT}+\mathrm{NPOINT}$ \\
\hline NPOINT & Number of wavelength points \\
\hline$V M I N$ & Minimum wavelength $(\mu \mathrm{m})$ \\
\hline$D E L V$ & -1.0 \\
\hline$F W H M$ & 0 \\
\hline$N P$ & Number of pressures \\
\hline$N T$ & Number of temperatures \\
\hline$N G$ & Number of gauss points \\
\hline$I D G A S 1$ & NEMESIS ID for gas species \\
\hline IS OGAS 1 & NEMESIS ID for gas isotopologue \\
\hline$G \_O R D S$ & List of G-oordinates gauss points \\
\hline G_WEIGHTS & List of weights for gauss points \\
\hline Blank & Float for 0 \\
\hline Blank & Float for 0 \\
\hline$p$ & List of pressures (bar) \\
\hline$t$ & List of temperatures $(\mathrm{K})$ \\
\hline bin_centres & Bin centres in wavelength $(\mu \mathrm{m})$ space \\
\hline kcoeff & $\begin{array}{c}K \text {-coefficient array }(\lambda, p, t, \text { gauss }) \\
\left(10^{20} \mathrm{~cm}^{2} / \text { molecule }\right)\end{array}$ \\
\hline
\end{tabular}

or nested sampling (PyMultiNest; Feroz \& Hobson 2008; Feroz et al. 2009, 2013; Buchner et al. 2014) algorithm. It is capable of simulating a range of planetary radiative transfer scenarios, including exoplanet transit, eclipse, and phase curve spectra, and nadir and limb sounding of Solar System atmospheres; it supports the inclusion of parametrised clouds, and for some geometries multiple scattering calculations can be performed. Table 5 gives a summary of the data contained within each NEMESIS (Irwin et al. 2008) $k$-table file, which are in binary format.

\section{4. petitRADTRANS}

petitRADTRANS (Mollière et al. 2019) is an open-source radiative transfer code for exoplanet spectra with the Python package and implemented retrieval examples available on the code website $^{6}$. petitRADTRANS can calculate emission and transmission spectra for cloudy and cloud-free atmospheres at high $\left(R=10^{6}\right)$ and low $(R=1000)$ resolution. The $R=1000$ branch of petitRADTRANS uses opacities in the form of $k$-tables, with a wavelength grid which differs slightly from that used in the $k$-tables and cross sections produced for the other retrieval codes. The $k$-tables for petitRADTRANS are in HDF5 format, for similar reasons to those mentioned in Sect. 4.1. Table 6 gives an overview of the data fields contained within the HDF5 $k$-tables for use in the petitRADTRANS retrieval code. The code available on the website has been updated to read directly from the $k$-tables in HDF5 format that are presented in this paper. This works in a plug-and-play fashion. See the petitRADTRANS website for more information.

\section{Line list sources and comments}

Tables 7-14 give details for all molecular data used in this work, with tables divided into groups ${ }^{7}$. Included in each table is the

\footnotetext{
6 https://petitradtrans.readthedocs.io/en/latest/

As at www. exomol.com
}

Table 6. Overview of the data fields contained within the HDF5 $k$-tables for use in the petitRADTRANS retrieval code.

\begin{tabular}{lc}
\hline \hline Field name & Description \\
\hline bin_centres & Bin centres in wavenumber $\left(\mathrm{cm}^{-1}\right)$ space \\
bin_edges & Bin edges in wavenumber $\left(\mathrm{cm}^{-1}\right)$ space \\
wlrange & Wavelength $(\mu \mathrm{m}): \min , \max$ \\
wnrange & Wavenumber $\left(\mathrm{cm}^{-1}\right)$ : min, max \\
samples & Gauss sampling points \\
weights & Weights for gauss sampling points \\
ngauss & Number of gauss sampling points \\
method & Description of sampling method \\
$k$ coeff & $K$-coefficient array $(p, t$, bin_centres, samples) \\
$k$ coeff.units & $K$-coefficient units (cm $/$ molecule) \\
$t$ & List of temperatures \\
$p$ & List of pressures \\
t.units & Units of temperature (K) \\
p.units & Units of pressure (bar) \\
mol_mass & Molecular mass in a.m.u. \\
Date_ID & ID for date of creation and version \\
DOI & Digital Online Identifier for line list \\
mol_name & Molecule name \\
key_iso_ll & ID for isotopologue and line list \\
\hline
\end{tabular}

source for the line list used for each molecule (this is what is considered to be the most complete, accurate, and up to date at the time of publication; the online ExoMol database will be updated to label the recommended line list for each molecular isotopologue if this changes); the associated minimum and maximum wavenumbers and wavelengths $\left(E_{l}, E_{u}, \lambda_{l}, \lambda_{u}\right.$, respectively); the number of lines in the line list; the number of levels in the line list; the temperature up to which the line list is considered complete $T_{\max }$; and an indication for further comments, if applicable, which can be found in Sect. 5.1.2. Table 15 gives the same information for select atomic species.

\subsection{Comments on tables}

\subsubsection{General comments}

The temperature of completeness for all the MoLLIST (Bernath 2020) molecules is assumed to be $5000 \mathrm{~K}$, although it is expected that most of these will not necessarily be complete, even at lower temperatures. Nevertheless, they are the best data currently available for these molecules. The opacities for these species will be updated in the future, if and when new line list data are obtained.

There are data available for some molecules not mentioned in the tables of this work, such as $\mathrm{PF}_{3}$ (Mant et al. 2019), which is currently only computed up to a low value of the rotational angular momentum quantum number, $J$. It is therefore incomplete in its current state, but can be computed if requested. Readers are encouraged to contact the ExoMol team if there are particular requests for molecular data not already computed.

As previously mentioned, a small number of molecules in the HITRAN (Gordon et al. 2017) database (HF, HCl, HBr, HI, $\mathrm{H}_{2}$ ) are considered applicable up to high temperatures of around 4000-5000 K (Li et al. 2013).

\subsubsection{Comments on individual species}

(1a) Work is underway for an updated ExoMol line list for $\mathrm{SiO}$ which will extend into the ultraviolet. The current line list only 
Table 7. Sources and properties of line list data used to compute the opacities presented in this work for metal oxides.

\begin{tabular}{|c|c|c|c|c|c|c|c|c|c|c|}
\hline Species & Line list & Ref. & $\begin{array}{c}E_{l} \\
\left(\mathrm{~cm}^{-1}\right)\end{array}$ & $\begin{array}{c}E_{u} \\
\left(\mathrm{~cm}^{-1}\right)\end{array}$ & $\begin{array}{c}\lambda_{l} \\
(\mu \mathrm{m})\end{array}$ & $\begin{array}{c}\lambda_{u} \\
(\mu \mathrm{m})\end{array}$ & Lines & Levels & $\begin{array}{c}T_{\max } \\
(\mathrm{K})\end{array}$ & Notes \\
\hline $\mathrm{AlO}$ & ExoMol ATP & Patrascu et al. (2015) & 100 & 35000 & 0.29 & 100 & 4.9 million & 94000 & 8000 & \\
\hline $\mathrm{CaO}$ & ExoMol VBATHY & Yurchenko et al. (2016) & 100 & 20000 & 0.5 & 100 & 28.4 million & 130000 & 5000 & \\
\hline $\mathrm{MgO}$ & ExoMol LiTY & Li et al. (2019) & 100 & 33000 & 0.3 & 100 & 72.8 million & 190000 & 5000 & \\
\hline $\mathrm{SiO}$ & ExoMol EBJT & Barton et al. (2013) & 100 & 6049 & 1.65 & 100 & 250000 & 24000 & 9000 & (1a) \\
\hline $\mathrm{TiO}$ & ExoMol TOTO & McKemmish et al. (2019) & 100 & 30000 & 0.33 & 100 & 30 million & 300000 & 5000 & \\
\hline VO & ExoMol VOMYT & McKemmish et al. (2016) & 100 & 35000 & 0.29 & 100 & 277 million & 640000 & 5000 & \\
\hline
\end{tabular}

Table 8. Sources and properties of line list data used to compute the opacities presented in this work for other oxides.

\begin{tabular}{|c|c|c|c|c|c|c|c|c|c|c|}
\hline Species & Line list & Ref. & $\begin{array}{c}E_{l} \\
\left(\mathrm{~cm}^{-1}\right)\end{array}$ & $\begin{array}{c}E_{u} \\
\left(\mathrm{~cm}^{-1}\right)\end{array}$ & $\begin{array}{c}\lambda_{l} \\
(\mu \mathrm{m})\end{array}$ & $\begin{array}{c}\lambda_{u} \\
(\mu \mathrm{m})\end{array}$ & Lines & Levels & $\begin{array}{c}T_{\max } \\
(\mathrm{K})\end{array}$ & Notes \\
\hline $\mathrm{CO}$ & Li 2015 & Li et al. (2015) & 100 & 23000 & 0.43 & 100 & 145000 & 6400 & 5000 & \\
\hline NO & HITEMP-2019 & Hargreaves et al. (2019) & 100 & 27000 & 0.37 & 100 & 1.1 million & - & 4000 & (1b) \\
\hline $\mathrm{O}_{2}$ & HITRAN & Gordon et al. (2017) & 100 & 6997 & 1.43 & 100 & 290000 & - & 296 & \\
\hline $\mathrm{PO}$ & ExoMol POPS & Prajapat et al. (2017) & 100 & 12000 & 0.83 & 100 & 2.1 million & 43000 & 5000 & \\
\hline
\end{tabular}

Table 9. Sources and properties of line list data used to compute the opacities presented in this work for triatomics.

\begin{tabular}{|c|c|c|c|c|c|c|c|c|c|c|}
\hline Species & Line list & Ref. & $\begin{array}{c}E_{l} \\
\left(\mathrm{~cm}^{-1}\right)\end{array}$ & $\begin{array}{c}E_{u} \\
\left(\mathrm{~cm}^{-1}\right)\end{array}$ & $\begin{array}{c}\lambda_{l} \\
(\mu \mathrm{m})\end{array}$ & $\begin{array}{c}\lambda_{u} \\
(\mu \mathrm{m})\end{array}$ & Lines & Levels & $\begin{array}{l}T_{\max } \\
(\mathrm{K})\end{array}$ & Notes \\
\hline $\mathrm{CO}_{2}$ & ExoMol UCL-4000 & Yurchenko et al. (2020a) & 100 & 20000 & 0.5 & 100 & 8 billion & 3.5 million & 4000 & (1c) \\
\hline $\mathrm{H}_{2} \mathrm{O}$ & $\begin{array}{c}\text { ExoMol } \\
\text { POKAZATEL }\end{array}$ & Polyansky et al. (2018) & 100 & 41200 & 0.24 & 100 & 6 billion & 800000 & 4000 & (1d) \\
\hline $\mathrm{H}_{2} \mathrm{~S}$ & ExoMol AYT2 & Azzam et al. (2016) & 100 & 11000 & 0.91 & 100 & 115 million & 220000 & 2000 & \\
\hline $\mathrm{HCN}$ & ExoMol Harris & Barber et al. (2014) & 100 & 18000 & 0.56 & 100 & 34.4 million & 170000 & 4000 & \\
\hline $\mathrm{N}_{2} \mathrm{O}$ & HITEMP-2019 & Hargreaves et al. (2019) & 100 & 12900 & 0.76 & 100 & 3.6 million & - & 1000 & \\
\hline $\mathrm{NO}_{2}$ & HITEMP-2019 & Hargreaves et al. (2019) & 100 & 4775 & 2.09 & 100 & 1.1 million & - & 1000 & \\
\hline $\mathrm{O}_{3}$ & HITRAN & Gordon et al. (2017) & 100 & 7000 & 1.43 & 100 & 290000 & - & 296 & \\
\hline $\mathrm{SiH}_{2}$ & ExoMol CATS & Clark et al. (2020) & 100 & 10000 & 1.00 & 100 & 310 million & 594000 & 2000 & \\
\hline $\mathrm{SiO}_{2}$ & ExoMol OYT3 & Owens et al. (2020) & 100 & 6000 & 1.67 & 100 & 32.9 billion & 5.7 million & 3000 & \\
\hline $\mathrm{SO}_{2}$ & ExoMol ExoAmes & Underwood et al. (2016a) & 100 & 8000 & 1.25 & 100 & 1.4 billion & 3.3 million & 2000 & \\
\hline
\end{tabular}

Table 10. Sources and properties of line list data used to compute the opacities presented in this work for metal hydrides.

\begin{tabular}{|c|c|c|c|c|c|c|c|c|c|c|}
\hline Species & Line list & Ref. & $\begin{array}{c}E_{l} \\
\left(\mathrm{~cm}^{-1}\right)\end{array}$ & $\begin{array}{c}E_{u} \\
\left(\mathrm{~cm}^{-1}\right)\end{array}$ & $\begin{array}{c}\lambda_{l} \\
(\mu \mathrm{m})\end{array}$ & $\begin{array}{c}\lambda_{u} \\
(\mu \mathrm{m})\end{array}$ & Lines & Levels & $\begin{array}{l}T_{\max } \\
(\mathrm{K})\end{array}$ & Notes \\
\hline $\mathrm{AlH}$ & ExoMol AlHambra & Yurchenko et al. (2018b) & 100 & 27000 & 0.37 & 100 & 36000 & 1500 & 5000 & \\
\hline $\mathrm{BeH}$ & $\begin{array}{c}\text { ExoMol } \\
\text { Darby-Lewis }\end{array}$ & Darby-Lewis et al. (2018) & 100 & 42000 & 0.24 & 100 & 590000 & 15000 & 2000 & \\
\hline $\mathrm{CaH}$ & MoLLIST & Li et al. (2012) & 100 & 22000 & 0.45 & 100 & 6000 & 914 & 5000 & \\
\hline $\mathrm{CrH}$ & MoLLIST & Burrows et al. (2002b) & 100 & 14500 & 0.69 & 100 & 13,800 & 1600 & 5000 & \\
\hline $\mathrm{FeH}$ & MoLLIST & Wende et al. (2010) & 100 & 15000 & 0.67 & 100 & 93000 & 3500 & 5000 & \\
\hline $\mathrm{LiH}$ & CLT & Coppola et al. (2011) & 100 & 20000 & 0.5 & 100 & 19000 & 1100 & 2000 & \\
\hline $\mathrm{MgH}$ & MoLLIST & Gharib-Nezhad et al. (2013) & 100 & 29000 & 0.34 & 100 & 14200 & 1300 & 2000 & (1e) \\
\hline $\mathrm{NaH}$ & ExoMol Rivlin & Rivlin et al. (2015) & 100 & 37000 & 0.27 & 100 & 80,000 & 3300 & 7000 & \\
\hline $\mathrm{ScH}$ & LYT & Lodi et al. (2015) & 100 & 15800 & 0.63 & 100 & 1.2 million & 8500 & 2000 & \\
\hline $\mathrm{TiH}$ & MoLLIST & Burrows et al. (2005) & 100 & 24000 & 0.42 & 100 & 200000 & 5800 & 5000 & \\
\hline
\end{tabular}

considers vibration-rotation transitions and so the current maximum wavenumber was set at $6049 \mathrm{~cm}^{-1}$. (1b) The HITEMP line list for NO includes data from the ExoMol NOname line list (Wong et al. 2017). (1c) The Ames line list (Huang et al. 2017) and the CDSD-4000 databank (Tashkun \& Perevalov 2011) are also available for $\mathrm{CO}_{2}$, as well as the HITEMP compilation (Rothman et al. 2010). (1d) The previous ExoMol line list for $\mathrm{H}_{2} \mathrm{O}$, BT2 (Barber et al. 2006), is only complete up to temperatures of $3000 \mathrm{~K}$, whereas the more accurate ExoMol POKAZATEL line list Polyansky et al. (2018) is complete up 
Table 11. Sources and properties of line list data used to compute the opacities presented in this work for other hydrides.

\begin{tabular}{|c|c|c|c|c|c|c|c|c|c|c|}
\hline Species & Line list & Ref. & $\begin{array}{c}E_{l} \\
\left(\mathrm{~cm}^{-1}\right)\end{array}$ & $\begin{array}{c}E_{u} \\
\left(\mathrm{~cm}^{-1}\right)\end{array}$ & $\begin{array}{c}\lambda_{l} \\
(\mu \mathrm{m})\end{array}$ & $\begin{array}{c}\lambda_{u} \\
(\mu \mathrm{m})\end{array}$ & Lines & Levels & $\begin{array}{l}T_{\max } \\
(\mathrm{K})\end{array}$ & Notes \\
\hline $\mathrm{CH}$ & MoLLIST & Masseron et al. (2014) & 100 & 39000 & 0.26 & 100 & 53000 & 2500 & 5000 & \\
\hline $\mathrm{HBr}$ & HITRAN & Li et al. (2013) & 100 & 16050 & 0.62 & 100 & 6070 & - & 5000 & \\
\hline $\mathrm{HCl}$ & HITRAN & Li et al. (2013) & 100 & 20230 & 0.49 & 100 & 8890 & - & 5000 & \\
\hline $\mathrm{HF}$ & HITRAN & Li et al. (2013) & 100 & 32350 & 0.31 & 100 & 8090 & - & 5000 & \\
\hline $\mathrm{HI}$ & HITRAN & Li et al. (2013) & 100 & 14000 & 0.71 & 100 & 3160 & - & 5000 & \\
\hline NH & MoLLIST & $\begin{array}{l}\text { Brooke et al. (2014a, 2015) } \\
\text { Fernando et al. (2018) }\end{array}$ & 100 & 16900 & 0.59 & 100 & 10400 & 740 & 5000 & \\
\hline $\mathrm{OH}$ & MoLLIST & $\begin{array}{l}\text { Brooke et al. (2016) } \\
\text { Yousefi et al. (2018) }\end{array}$ & 100 & 43400 & 0.23 & 100 & 54000 & 1900 & 5000 & \\
\hline $\mathrm{PH}$ & ExoMol LaTY & Langleben et al. (2019) & 100 & 24500 & 0.41 & 100 & 64800 & 2500 & 4000 & \\
\hline $\mathrm{SiH}$ & ExoMol SiGHTLY & Yurchenko et al. (2018c) & 100 & 31000 & 0.32 & 100 & 1.7 million & 11800 & 5000 & \\
\hline $\mathrm{SH}$ & ExoMol GYT & Gorman et al. (2019) & 100 & 39000 & 0.26 & 100 & 572145 & 7686 & 5000 & \\
\hline
\end{tabular}

Table 12. Sources and properties of line list data used to compute the opacities presented in this work for other diatomics.

\begin{tabular}{|c|c|c|c|c|c|c|c|c|c|c|}
\hline Species & Line list & Ref. & $\begin{array}{c}E_{l} \\
\left(\mathrm{~cm}^{-1}\right)\end{array}$ & $\begin{array}{c}E_{u} \\
\left(\mathrm{~cm}^{-1}\right)\end{array}$ & $\begin{array}{c}\lambda_{l} \\
(\mu \mathrm{m})\end{array}$ & $\begin{array}{c}\lambda_{u} \\
(\mu \mathrm{m})\end{array}$ & Lines & Levels & $\begin{array}{l}T_{\max } \\
(\mathrm{K})\end{array}$ & Notes \\
\hline $\mathrm{AlCl}$ & MoLLIST & Yousefi \& Bernath (2018) & 100 & 2350 & 4.26 & 100 & 20200 & 2400 & 5000 & \\
\hline $\mathrm{AlF}$ & MoLLIST & Yousefi \& Bernath (2018) & 100 & 3880 & 2.58 & 100 & 40500 & 2420 & 5000 & \\
\hline $\mathrm{C}_{2}$ & ExoMol 8states & Yurchenko et al. (2018d) & 100 & 48660 & 0.21 & 100 & 6.1 million & 44190 & 5000 & \\
\hline $\mathrm{CaF}$ & MoLLIST & Hou \& Bernath (2018) & 100 & 5580 & 1.79 & 100 & 14800 & 1360 & 5000 & \\
\hline $\mathrm{CN}$ & MoLLIST & Brooke et al. (2014b) & 100 & 44200 & 0.23 & 100 & 195000 & 7700 & 5000 & \\
\hline $\mathrm{CP}$ & MoLLIST & Ram et al. (2014) & 100 & 15000 & 0.67 & 100 & 28700 & 2100 & 5000 & \\
\hline $\mathrm{CS}$ & ExoMol JnK & Paulose et al. (2015) & 100 & 11000 & 0.91 & 100 & 199000 & 11500 & 3000 & \\
\hline $\mathrm{H}_{2}$ & RACPPK & Roueff et al. (2019) & 100 & 36000 & 0.28 & 100 & 4700 & 300 & 5000 & \\
\hline $\mathrm{KCl}$ & ExoMol Barton & Barton et al. (2014) & 100 & 2900 & 3.45 & 100 & 1.3 million & 60700 & 3000 & \\
\hline $\mathrm{KF}$ & MoLLIST & Frohman et al. (2016) & 100 & 4000 & 2.49 & 100 & 10500 & 1060 & 5000 & \\
\hline $\mathrm{LiCl}$ & MoLLIST & Bittner \& Bernath (2018) & 100 & 4840 & 2.07 & 100 & 26200 & 2400 & 5000 & \\
\hline $\mathrm{LiF}$ & MoLLIST & Bittner \& Bernath (2018) & 100 & 1810 & 5.52 & 100 & 10600 & 2400 & 5000 & \\
\hline $\mathrm{MgF}$ & MoLLIST & Hou \& Bernath (2017) & 100 & 5470 & 1.83 & 100 & 8100 & 900 & 5000 & \\
\hline $\mathrm{NaCl}$ & ExoMol Barton & Barton et al. (2014) & 100 & 2500 & 4.00 & 100 & 703000 & 49,000 & 3000 & \\
\hline $\mathrm{NaF}$ & MoLLIST & Frohman et al. (2016) & 100 & 4990 & 2.01 & 100 & 7900 & 840 & 5000 & \\
\hline NS & ExoMol SNaSH & Yurchenko et al. (2018e) & 100 & 38420 & 0.26 & 100 & 3.2 million & 31500 & 5000 & \\
\hline $\mathrm{PN}$ & ExoMol YYLT & Yorke et al. (2014) & 100 & 6500 & 1.54 & 100 & 140000 & 14000 & 5000 & \\
\hline PS & ExoMol POPS & Prajapat et al. (2017) & 100 & 36700 & 0.27 & 100 & 30.4 million & 226000 & 5000 & \\
\hline $\mathrm{SiS}$ & ExoMol UCTY & Upadhyay et al. (2018) & 100 & 3700 & 2.70 & 100 & 91600 & 10000 & 5000 & \\
\hline
\end{tabular}

to $4000 \mathrm{~K}$. (1e) There is also a line list for MgH from ExoMol Yadin (Yadin et al. 2012). However, since it only covers the ground electronic $X^{2} \Sigma^{+}$state, and so is less complete than the more recent MoLLIST line list of Gharib-Nezhad et al. (2013), we use the latter. (1f) Previous to the ExoMol aCeTY line list of Chubb et al. (2020b), the main sources of data for acetylene were from HITRAN (Gordon et al. 2017) and ASD1000 (Lyulin \& Perevalov 2017). The data from HITRAN are only applicable for studies performed at room temperature, and were shown in Chubb et al. (2020b) to be inadequate for hightemperature applications. ASD-1000 was a vast improvement, although there does seem to be opacity missing from some of the hot bands when compared to ExoMol aCeTY in Chubb et al. (2020b). (1g) The previous ExoMol line list for $\mathrm{CH}_{4}$, called 10-10 (Yurchenko \& Tennyson 2014), is only complete up to $1500 \mathrm{~K}$. The updated 34-10 line list is therefore recommended instead. Future updates of the database will investigate using data for methane based on recent line lists from either TheoReTs
(Rey et al. 2017) or HITEMP (Hargreaves et al. 2020); these are currently expected to be more accurate when considering high-resolution applications. For low-resolution applications, we expect the quality of the ExoMol line list used here to be sufficient, particularly because completeness is more important than accuracy at lower resolutions (Yurchenko et al. 2014). (1h) The energy states from Coppola et al. (2011) are used in the Amaral et al. (2019) line list for $\mathrm{HD}^{+}$. (1i) The energy states from Engel et al. (2005) are used in the Amaral et al. (2019) line list for $\mathrm{HeH}^{+}$. (1j) The pressure and temperature broadened profiles for the resonance doublets of $\mathrm{Na}$ and $\mathrm{K}$ are computed using Allard et al. (2016, 2019). See Sect. 3.2.3 for a discussion on the broadening profiles of these atoms.

\subsection{Isotopologues}

For the majority of species, we provide the opacities for the main isotopologue only, or separate opacity files for the other 
Table 13. Sources and properties of line list data used to compute the opacities presented in this work for larger molecules.

\begin{tabular}{|c|c|c|c|c|c|c|c|c|c|c|}
\hline Species & Line list & Ref. & $\begin{array}{c}E_{l} \\
\left(\mathrm{~cm}^{-1}\right)\end{array}$ & $\begin{array}{c}E_{u} \\
\left(\mathrm{~cm}^{-1}\right)\end{array}$ & $\begin{array}{c}\lambda_{l} \\
(\mu \mathrm{m})\end{array}$ & $\begin{array}{c}\lambda_{u} \\
(\mu \mathrm{m})\end{array}$ & Lines & Levels & $\begin{array}{c}T_{\max } \\
(\mathrm{K})\end{array}$ & Notes \\
\hline $\mathrm{AsH}_{3}$ & ExoMol CYT18 & Coles et al. (2019a) & 100 & 7000 & 1.43 & 100 & 3.6 million & 4.3 million & 296 & \\
\hline $\mathrm{C}_{2} \mathrm{H}_{2}$ & ExoMol aCeTY & Chubb et al. (2020b) & 100 & 10000 & 1.00 & 100 & 4.3 billion & 5.2 million & 2200 & (1f) \\
\hline $\mathrm{C}_{2} \mathrm{H}_{4}$ & ExoMol MaYTY & Mant et al. (2018) & 100 & 7100 & 1.41 & 100 & 50 billion & 45 million & 700 & \\
\hline $\mathrm{CH}_{3}$ & ExoMol AYYJ & Adam et al. (2019) & 100 & 10000 & 1.00 & 100 & 2.1 billion & 9.1 million & 1500 & \\
\hline $\mathrm{CH}_{3} \mathrm{Cl}$ & ExoMol OYT & Owens et al. (2018a) & 100 & 6400 & 1.56 & 100 & 166 billion & 10.2 million & 1200 & \\
\hline $\mathrm{CH}_{3} \mathrm{~F}$ & ExoMol OYKYT & Owens et al. (2018b) & 100 & 4700 & 2.13 & 100 & 1.4 billion & 3.5 million & 300 & \\
\hline $\mathrm{CH}_{4}$ & ExoMol 34to10 & Yurchenko et al. (2017a) & 100 & 18000 & 0.56 & 100 & 34 billion & 8.2 million & 2000 & $(1 \mathrm{~g})$ \\
\hline $\mathrm{H}_{2} \mathrm{O}_{2}$ & ExoMol APTY & Al-Refaie et al. (2016) & 100 & 6000 & 1.67 & 100 & 10 billion & 7.6 million & 1250 & \\
\hline $\mathrm{H}_{2} \mathrm{CO}$ & ExoMol AYTY & Al-Refaie et al. (2015) & 100 & 10100 & 0.99 & 100 & 10 billion & 10.3 million & 1500 & \\
\hline $\mathrm{HNO}_{3}$ & ExoMol AIJS & Pavlyuchko et al. (2015) & 100 & 7100 & 1.41 & 100 & 7 billion & 17.5 million & 500 & \\
\hline $\mathrm{NH}_{3}$ & ExoMol CoYuTe & Coles et al. (2019b) & 100 & 20000 & 0.5 & 100 & 16.9 billion & 5.1 million & 1500 & \\
\hline $\begin{array}{l}\mathrm{P}_{2} \mathrm{H}_{2} \\
\text { (cis) }\end{array}$ & OY-Cis & Owens \& Yurchenko (2019) & 100 & 6000 & 1.67 & 100 & 5.9 billion & 6 million & 300 & \\
\hline $\begin{array}{l}\mathrm{P}_{2} \mathrm{H}_{2} \\
\text { (trans) }\end{array}$ & OY-Trans & Owens \& Yurchenko (2019) & 100 & 6000 & 1.67 & 100 & 5.3 billion & 5.9 million & 300 & \\
\hline $\mathrm{PH}_{3}$ & ExoMol SAITY & Sousa-Silva et al. (2015) & 100 & 10000 & 1.00 & 100 & 16.8 billion & 9.8 million & 1500 & \\
\hline $\mathrm{SiH}_{4}$ & ExoMol OY2T & Owens et al. (2017) & 100 & 5000 & 2.00 & 100 & 62.7 billion & 7.1 million & 1200 & \\
\hline $\mathrm{SO}_{3}$ & ExoMol UYT2 & Underwood et al. (2016b) & 100 & 5000 & 2.00 & 100 & 21 billion & 18.5 million & 800 & \\
\hline
\end{tabular}

Table 14. Sources and properties of line list data used to compute the opacities presented in this work for ions.

\begin{tabular}{|c|c|c|c|c|c|c|c|c|c|c|}
\hline Species & Line list & Ref & $\begin{array}{c}\mathrm{E}_{l} \\
\left(\mathrm{~cm}^{-1}\right)\end{array}$ & $\begin{array}{c}\mathrm{E}_{u} \\
\left(\mathrm{~cm}^{-1}\right)\end{array}$ & $\begin{array}{c}\lambda_{l} \\
(\mu \mathrm{m})\end{array}$ & $\begin{array}{c}\lambda_{u} \\
(\mu \mathrm{m})\end{array}$ & Lines & Levels & $\begin{array}{l}\mathrm{T}_{\max } \\
(\mathrm{K})\end{array}$ & Notes \\
\hline $\mathrm{H}_{3}{ }^{+}$ & ExoMol MiZATeP & Mizus et al. (2017) & 100 & 25000 & 0.4 & 100 & 127.5 million & 159000 & 5000 & \\
\hline $\mathrm{H}_{3} \mathrm{O}^{+}$ & ExoMol eXeL & Yurchenko et al. (2020b) & 100 & 10000 & 1.00 & 100 & 2.1 billion & 1.2 million & 1500 & \\
\hline $\mathrm{HD}^{+}$ & ADJSAAM & Amaral et al. (2019) & 100 & 21500 & 0.47 & 100 & 10300 & 640 & 4000 & (1h) \\
\hline $\mathrm{HeH}^{+}$ & ADJSAAM & Amaral et al. (2019) & 100 & 14900 & 0.67 & 100 & 1400 & 180 & 4000 & (1i) \\
\hline $\mathrm{LiH}^{+}$ & CLT & Coppola et al. (2011) & 100 & 920 & 10.87 & 100 & 330 & 75 & 2000 & \\
\hline $\mathrm{OH}^{+}$ & MoLLIST & Hodges \& Bernath (2017) & 100 & 30300 & 0.33 & 100 & 12000 & 820 & 5000 & \\
\hline
\end{tabular}

Table 15. Sources and properties of line list data used to compute the opacities presented in this work for atoms.

\begin{tabular}{lcccccccccc}
\hline \hline Species & Line list & Ref. & $\begin{array}{c}E_{l} \\
\left(\mathrm{~cm}^{-1}\right)\end{array}$ & $\begin{array}{c}E_{u} \\
\left(\mathrm{~cm}^{-1}\right)\end{array}$ & $\begin{array}{c}\lambda_{l} \\
(\mu \mathrm{m})\end{array}$ & $\begin{array}{c}\lambda_{u} \\
(\mu \mathrm{m})\end{array}$ & Lines & Levels & $\begin{array}{c}T_{\max } \\
(\mathrm{K})\end{array}$ & Notes \\
\hline $\mathrm{K}$ & NIST & Kramida et al. (2013) & 100 & 35000 & 0.29 & 100 & 186 & 188 & 5000 & $(1 \mathrm{j})$ \\
$\mathrm{Na}$ & NIST & Kramida et al. (2013) & 100 & 42000 & 0.24 & 100 & 523 & 117 & 5000 & $(1 \mathrm{j})$ \\
\hline
\end{tabular}

isotopologues. However, for some species it is important to take natural abundances into account (see, e.g. Coursey et al. 2015). We therefore provide opacity files combined at natural abundances for the species listed in Table 16, as well as the separate isotopologue opacities.

The species included in this table may be updated if new isotopologue line lists become available (line lists can be extended to different isotopologues using the method outlined in Polyansky et al. 2017, with line positions approaching experimental accuracy for species such as $\mathrm{H}_{2} \mathrm{O}$ ). A summary of the number of isotopologues available for various line lists can be found in Tennyson \& Yurchenko (2018).

\section{Visible and UV}

The wavelength regions covered by the data for each molecule should be carefully noted (see Tables 7-14); many of the diatomic molecules (such as $\mathrm{PO}, \mathrm{SiO}, \mathrm{CrH}, \mathrm{FeH}, \mathrm{NH}, \mathrm{PN}, \mathrm{KCl}$,
$\mathrm{NaCl}, \mathrm{LiCl}, \mathrm{CS}, \mathrm{CP}, \mathrm{AlCl}, \mathrm{AlF}, \mathrm{KF}, \mathrm{LiF}, \mathrm{CaF}, \mathrm{MgF}$ ) are not covered for wavelengths short of $0.67 \mu \mathrm{m}$, even though they are expected to have opacity in this region. Using our opacities for such species to characterise observations that span beyond this region is therefore not advised, as it could give the impression that the opacity suddenly drops off at the wavelength at which the data ends, which in practise would not be true. It is therefore desirable to have these opacities computed to higher energies (corresponding to lower wavelengths). At present, some data are available in these regions for certain molecules. The ExoMol project is planning further work on this problem which will need to consider bound-free (photodissociation) as well as bound-bound processes.

\section{The ExoMolOP database}

The ExoMolOP database comprises opacity data for over 80 species, details of which can be found in Tables 7-15. The data 
Table 16. Species where the opacities are combined according to natural elemental abundances.

\begin{tabular}{|c|c|c|c|}
\hline Species & Iso & NA $(\%)$ & MM (a.m.u.) \\
\hline $\mathrm{TiO}$ & ${ }^{48} \mathrm{Ti}^{16} \mathrm{O}$ & 73.7 & 63.94 \\
\hline $\mathrm{TiO}$ & ${ }^{46} \mathrm{Ti}^{16} \mathrm{O}$ & 8.3 & 61.94 \\
\hline $\mathrm{TiO}$ & ${ }^{47} \mathrm{Ti}^{16} \mathrm{O}$ & 7.4 & 62.94 \\
\hline $\mathrm{TiO}$ & ${ }^{49} \mathrm{Ti}^{16} \mathrm{O}$ & 5.4 & 64.94 \\
\hline $\mathrm{TiO}$ & ${ }^{50} \mathrm{Ti}^{16} \mathrm{O}$ & 5.2 & 65.94 \\
\hline $\mathrm{CO}$ & ${ }^{12} \mathrm{C}^{16} \mathrm{O}$ & 98.7 & 27.99 \\
\hline $\mathrm{CO}$ & ${ }^{13} \mathrm{C}^{16} \mathrm{O}$ & 1.1 & 28.99 \\
\hline $\mathrm{CO}$ & ${ }^{12} \mathrm{C}^{18} \mathrm{O}$ & $2.0 \times 10^{-3}$ & 29.99 \\
\hline $\mathrm{CO}$ & ${ }^{12} \mathrm{C}^{17} \mathrm{O}$ & $3.7 \times 10^{-4}$ & 28.99 \\
\hline $\mathrm{CO}$ & ${ }^{13} \mathrm{C}^{18} \mathrm{O}$ & $2.2 \times 10^{-5}$ & 31.00 \\
\hline $\mathrm{CO}$ & ${ }^{13} \mathrm{C}^{17} \mathrm{O}$ & $4.1 \times 10^{-6}$ & 30.00 \\
\hline $\mathrm{HBr}$ & $\mathrm{H}^{79} \mathrm{Br}$ & 50.7 & 79.93 \\
\hline $\mathrm{HBr}$ & $\mathrm{H}^{81} \mathrm{Br}$ & 49.3 & 81.92 \\
\hline $\mathrm{HBr}$ & $\mathrm{D}^{79} \mathrm{Br}$ & $7.9 \times 10^{-3}$ & 80.93 \\
\hline $\mathrm{HBr}$ & $\mathrm{D}^{81} \mathrm{Br}$ & $7.7 \times 10^{-3}$ & 82.93 \\
\hline $\mathrm{HCl}$ & $\mathrm{H}^{35} \mathrm{Cl}$ & 75.8 & 35.98 \\
\hline $\mathrm{HCl}$ & $\mathrm{H}^{37} \mathrm{Cl}$ & 24.2 & 37.97 \\
\hline $\mathrm{HCl}$ & $\mathrm{D}^{35} \mathrm{Cl}$ & $1.2 \times 10^{-2}$ & 36.98 \\
\hline $\mathrm{HCl}$ & $\mathrm{D}^{37} \mathrm{Cl}$ & $3.8 \times 10^{-3}$ & 38.98 \\
\hline $\mathrm{CH}_{3} \mathrm{Cl}$ & ${ }^{12} \mathrm{CH}_{3}{ }^{35} \mathrm{Cl}$ & 74.9 & 49.99 \\
\hline $\mathrm{CH}_{3} \mathrm{Cl}$ & ${ }^{12} \mathrm{CH}_{3}{ }^{37} \mathrm{Cl}$ & 23.9 & 51.99 \\
\hline $\mathrm{KCl}$ & ${ }^{39} \mathrm{~K}^{35} \mathrm{Cl}$ & 70.6 & 73.93 \\
\hline $\mathrm{KCl}$ & ${ }^{39} \mathrm{~K}^{37} \mathrm{Cl}$ & 22.6 & 75.93 \\
\hline $\mathrm{KCl}$ & ${ }^{41} \mathrm{~K}^{35} \mathrm{Cl}$ & 5.1 & 75.93 \\
\hline $\mathrm{KCl}$ & ${ }^{41} \mathrm{~K}^{37} \mathrm{Cl}$ & 1.6 & 77.92 \\
\hline $\mathrm{NaCl}$ & ${ }^{23} \mathrm{Na}^{35} \mathrm{Cl}$ & 75.8 & 57.96 \\
\hline $\mathrm{NaCl}$ & ${ }^{23} \mathrm{Na}^{37} \mathrm{Cl}$ & 24.2 & 59.96 \\
\hline $\mathrm{LiCl}$ & ${ }^{7} \mathrm{Li}^{35} \mathrm{Cl}$ & 70.0 & 41.98 \\
\hline $\mathrm{LiCl}$ & ${ }^{7} \mathrm{Li}^{37} \mathrm{Cl}$ & 22.4 & 43.98 \\
\hline $\mathrm{LiCl}$ & ${ }^{6} \mathrm{Li}^{35} \mathrm{Cl}$ & 5.8 & 40.98 \\
\hline $\mathrm{LiCl}$ & ${ }^{6} \mathrm{Li}^{37} \mathrm{Cl}$ & 1.8 & 42.98 \\
\hline $\mathrm{AlCl}$ & ${ }^{27} \mathrm{Al}^{35} \mathrm{Cl}$ & 75.8 & 61.95 \\
\hline $\mathrm{AlCl}$ & ${ }^{27} \mathrm{Al}^{37} \mathrm{Cl}$ & 24.2 & 63.95 \\
\hline $\mathrm{MgO}$ & ${ }^{24} \mathrm{Mg}^{16} \mathrm{O}$ & 78.8 & 39.98 \\
\hline $\mathrm{MgO}$ & ${ }^{25} \mathrm{Mg}^{16} \mathrm{O}$ & 9.9 & 40.98 \\
\hline $\mathrm{MgO}$ & ${ }^{26} \mathrm{Mg}^{16} \mathrm{O}$ & 10.9 & 41.98 \\
\hline $\mathrm{MgO}$ & ${ }^{24} \mathrm{Mg}^{17} \mathrm{O}$ & $3.0 \times 10^{-2}$ & 40.98 \\
\hline $\mathrm{MgO}$ & ${ }^{24} \mathrm{Mg}^{18} \mathrm{O}$ & 0.2 & 41.98 \\
\hline $\mathrm{MgH}$ & ${ }^{24} \mathrm{MgH}$ & 79.0 & 24.99 \\
\hline $\mathrm{MgH}$ & ${ }^{25} \mathrm{MgH}$ & 10.0 & 25.99 \\
\hline $\mathrm{MgH}$ & ${ }^{26} \mathrm{MgH}$ & 11.0 & 26.99 \\
\hline $\mathrm{MgF}$ & ${ }^{24} \mathrm{Mg}^{19} \mathrm{~F}$ & 79.0 & 42.98 \\
\hline $\mathrm{MgF}$ & ${ }^{25} \mathrm{Mg}^{19} \mathrm{~F}$ & 10.0 & 43.98 \\
\hline $\mathrm{MgF}$ & ${ }^{26} \mathrm{Mg}^{19} \mathrm{~F}$ & 11.0 & 44.98 \\
\hline
\end{tabular}

Notes. NA is the natural abundance, and MM is the molecular mass of each isotopologue.

are formatted in different ways for four different exoplanet atmosphere retrieval codes; ARCiS, TauREx, NEMESIS and petitRADTRANS (see Sect. 4), and include cross sections (at $R=$ $\frac{\lambda}{\Delta \lambda}=15000$ ) and $k$-tables (at $R=1000$ ) for the $0.3-50 \mu \mathrm{m}$ wavelength region. Voigt profiles are used to represent the broadening of molecular lines, using the broadening parameters detailed in Tables A.1-A.11, with line wings computed to 500 Voigt widths from the line centres. The pressure and temperature broadened profiles for the atomic resonance doublets of $\mathrm{Na}$ and $\mathrm{K}$ are computed using the tables of Allard et al. $(2016,2019)$.

\subsection{Opacity data location}

The opacity database is available online ${ }^{8}$. Opacity files can be downloaded online ${ }^{9}$ and used directly for four retrieval codes; ARCiS, TauREx, NEMESIS and petitRADTRANS, but are intended to be sufficiently easy to manipulate for general use also.

The data will be fully integrated into ExoMol and will form part of the 2020 release which has just been completed (Tennyson et al. 2020). The opacity cross sections and $k$-tables will also be made available via the virtual atomic and molecular data centre (VAMDC) portal (Dubernet et al. 2010; Dubernet \& Antony 2016).

\subsection{Keeping opacity data up to date}

The ExoMol application programming interface (API) is described in Tennyson et al. (2016), with an associated master definition file, ExoMol.all, available online ${ }^{10}$. The "def" files are accessed at URLs of the form www. exomol. com $/ \mathrm{db} /<\mathrm{mole}$ cule $>/<$ iso-slug $>/<$ dataset-name $>/<$ iso-slug $>$ __ $<$ da taset-name $>$. def, with the latest dataset name for a particular molecule given in ExoMol.all. This allows for automated updates of ExoMol data to be converted to opacity (cross section and $k$-table) data using the HTTP WGET formalism. We use the same format as described in the ExoMol master definition files for our cross section and $k$-table file naming (also key_iso_ll within the files; see Sect. 4). Opacity files can therefore be downloaded using a URL in the following form for each of the four retrieval codes:

- www.exomol.com/db/<molecule $>/<$ iso-slug $>$ $/<$ dataset-name $>/<$ iso-slug $>$ __ $<$ dataset-name $>$ .R1000_0.3-50mu.ktable.NEMESIS.kta

- www.exomol.com/db/<molecule $>/<$ iso-slug $>$ $/<$ dataset-name $>/<$ iso-slug $>$ __ $<$ dataset-name $>$ .R15000_0.3-50mu.xsec.TauREx.h5

- www.exomol.com/db/<molecule $>/<$ iso-slug $>$ $/<$ dataset-name $>/<$ iso-slug $>$ __<dataset-name $>$ .R1000_0.3-50mu.ktable.ARCiS.fits.gz

- www.exomol.com/db/<molecule $>/<$ iso-slug $>$ $/<$ dataset-name $>/<$ iso-slug $>$ __ $<$ dataset-name $>$ .R1000_0.3-50mu.ktable.petitRADTRANS.h5

Most of the files produced have a version ID field contained within (Date_ID), so future updates can be tracked. For example, if the cross sections or $k$-tables are recomputed with improved broadening parameters, new versions will be released. It should also be noted that studies which investigate the computational feasibility versus retrieval accuracy of both $k$-tables and cross sections were made before some more recent computational improvements, such as more widespread use of GPUs (see, e.g. Al-Refaie et al. 2019), and similar investigations may be beneficial. Future datasets may therefore be computed at higher $R=\frac{\lambda}{\Delta \lambda}$ to reflect requirements. It should also be noted that data from high-resolution observations require line-by-line integrated opacities for analysis, as discussed below, which are

\footnotetext{
8 WWW . exomol.com

9 WWW.exomol.com/data/data-types/opacity/

10 wWW. exomol.com/exomol.all
} 
typically only computed in the wavelength region necessary to match observations. Such opacities are not included in the present database, but may be added in the future. For these reasons, users of the ExoMolOP database are strongly advised to reference the version of the opacities used in publications, along with the associated line list.

\section{High-resolution opacity requirements}

It is stressed that this database is not intended for high-resolution applications. It would be beneficial to compute a series of very high-resolution cross sections, but restricted only to wavelength regions necessary to match available observational data for cross-correlation studies; see, for example, de Kok et al. (2014), Hawker et al. (2018), Mollière \& Snellen (2019), Webb et al. (2020). Currently, only a small sample of the line lists which are detailed in Tables 7-14 of Sect. 5 are suitable for use in highresolution applications, with cross sections typically required to be sampled to a resolution of at least $R=\frac{\lambda}{\Delta \lambda}=100000$. This includes those molecules which are found in the HITEMP (Rothman et al. 2010), HITRAN (Gordon et al. 2017), or MoLLIST (Bernath 2020) databases, and only those in the ExoMol (Tennyson \& Yurchenko 2012; Tennyson et al. 2016) database which have been "MARVELised". MARVEL (measured active vibration-rotation energy levels) is a prodecure whereby transition wavenumbers from all available laboratory experiments are analysed together to produce a list of experimentally determined energy levels (Furtenbacher et al. 2007; Tóbiás et al. 2019). These empirical energies are then subsequently included in the MARVELised ExoMol line lists to improve their accuracy (see, e.g. Chubb et al. 2018, 2020b; McKemmish et al. 2020). As only a subsection of the energy levels, and therefore transitions, which are included in an ExoMol line list will have been MARVELised, the new ExoMol data format includes an uncertainty column in the .states file (Tennyson et al. 2020). This gives an indication of the reliability of an individual energy level, and therefore of all transitions which involve this level. A set of high-resolution cross sections for six molecular species has recently been made publicly available by Gandhi et al. (2020).

\section{Conclusion}

In this work we present a publicly available database of opacity cross sections and $k$-tables for molecules of astrophysical interest, ExoMolOP, which is primarily based on the latest line list data from the ExoMol (Tennyson \& Yurchenko 2012; Tennyson et al. 2016), HITEMP (Rothman et al. 2010; Hargreaves et al. 2019) and MoLLIST (Bernath 2020) databases. These data are generally suitable for characterising high-temperature exoplanet or cool stellar atmospheres, and have been computed at a variety of pressures and temperatures, with a few molecules included at room temperature only from the HITRAN database. The data are formatted in different ways for four different exoplanet atmosphere retrieval codes; ARCiS (Min et al. 2020), TauREx (Waldmann et al. 2015a,b; Al-Refaie et al. 2019), NEMESIS (Irwin et al. 2008), and petitRADTRANS (Mollière et al. 2019). Opacity data for $\mathrm{Na}$ and $\mathrm{K}$ are also included using line-list data from the NIST (Kramida et al. 2013) database and the broadening scheme of Allard et al. (2016, 2019).

New opacities will be added to the ExoMolOP database as new or updated line lists become available. Updating an existing line list may include the MARVEL procedure (which can lead to noticeable differences in line positions, even at low resolution). As previously mentioned, the current ExoMolOP database is not suitable for high-resolution cross-correlation studies, but high-resolution opacities may be provided in the future alongside the current database. There are some opacities for high-resolution Doppler shift studies currently available from Gandhi et al. (2020). For the current release of the ExoMolOP database, a number of assumptions related to the broadening parameters were made, which can clearly be improved upon. The upcoming ExoMolHD project will focus on this explicitly for the next release of the database and will make use of advances in molecular broadening and line-shape theory (Stolarczyk et al. 2020; Hartmann et al. 2018). Even though the broadening parameters used in this work can clearly be improved, they are generally considered adequate for low-resolution retrieval studies. However, particular care should be taken when using these opacities in regimes where the broadening parameters may have more effect, such as for low-pressure and low-temperature environments.

Currently, only $\mathrm{H}_{2}$ and $\mathrm{He}$ parameters have been used, as the primary intention is for the characterisation of Hot Jupiter exoplanets. However, future releases of the database will extend to other types of broadener, such as self-broadening, $\mathrm{N}_{2}, \mathrm{CO}_{2}$, and air, which are thought to be important in other types of planet, such as mini-Neptunes or super Earths. It is thought that, for some molecules in particular, for example $\mathrm{H}_{2} \mathrm{O}$, the differences between self-broadening parameters can be around seven times larger than the $\mathrm{H}_{2} / \mathrm{He}$ parameters (Brown et al. 2005; Ptashnik et al. 2016; Gharib-Nezhad \& Line 2019).

There are often different versions of computed line lists for one molecule, some with quite stark differences. Users of the ExoMolOP database are strongly urged to include citations to the line list relating to the opacities used in their publications. We include a bibtex file with the citations to all opacities included as part of the supplementary data to this work.

Acknowledgements. This project has received funding from the European Union's Horizon 2020 Research and Innovation Programme, under Grant Agreement 776403, and from the European Research Council (ERC) under the European Union's Horizon 2020 research and innovation programme under grant agreement No 758892, ExoAI. P.M. acknowledges support from the European Research Council under the European Union's Horizon 2020 research and innovation program under grant agreement No. 832428. J.T. and S.Y. thank the STFC Project No. ST/R000476/1. We gratefully acknowledge Cambridge Service for Data Driven Discovery (CSD3), part of which is operated by the University of Cambridge Research Computing on behalf of the STFC DiRAC HPC Facility (www.dirac.ac.uk). The DiRAC component of CSD3 was funded by BEIS capital funding via STFC capital grants ST/P002307/1 and ST/R002452/1 and STFC operations grant ST/R00689X/1. DiRAC is part of the National eInfrastructure. We would like to thank Nicole Allard for providing all the data necessary to compute the broadening profiles for the $\mathrm{Na}$ and $\mathrm{K}$ resonance doublets. We thank the reviewer for their comments to improve the manuscript.

\section{References}

Adam, A. Y., Yachmenev, A., Yurchenko, S. N., \& Jensen, P. 2019, J. Phys. Chem. A, 123, 4755

Allard, N. F., Kielkopf, J. F., \& Allard, F. 2007, Eur. Phys. J. D, 44, 507

Allard, F., Homeier, D., \& Freytag, B. 2012, Philos. Trans. R. Soc. A: Math. Phys. Eng. Sci., 370, 2765

Allard, N. F., Spiegelman, F., \& Kielkopf, J. F. 2016, A\&A, 589, A21

Allard, N. F., Spiegelman, F., Leininger, T., \& Mollière, P. 2019, A\&A, 628, A120

Al-Refaie, A. F., Yurchenko, S. N., Yachmenev, A., \& Tennyson, J. 2015, MNRAS, 448, 1704

Al-Refaie, A. F., Polyansky, O. L., Ovsyannikov, R. I., Tennyson, J., \& Yurchenko, S. N. 2016, MNRAS, 461, 1012

Al-Refaie, A. F., Changeat, Q., Waldmann, I. P., \& Tinetti, G. 2019, ArXiv eprints [arXiv:1912.07759]

Amaral, P. H. R., Diniz, L. G., Jones, K. A., et al. 2019, ApJ, 878, 95

Amundsen, D. S., Baraffe, I., Tremblin, P., et al. 2014, A\&A, 564, A59 
Azzam, A. A. A., Yurchenko, S. N., Tennyson, J., \& Naumenko, O. V. 2016, MNRAS, 460, 4063

Ba, Y. A., Wenger, C., Surleau, R., et al. 2013, J. Quant. Spectr. Rad. Transf., 130,62

Badreddine, K., El-Kork, N., \& Korek, M. 2013, J. Mod. Phys., 4, 82

Barber, R. J., Tennyson, J., Harris, G. J., \& Tolchenov, R. N. 2006, MNRAS, 368,1087

Barber, R. J., Strange, J. K., Hill, C., et al. 2014, MNRAS, 437, 1828

Barstow, J. K., Aigrain, S., Irwin, P. G. J., \& Sing, D. K. 2016, ApJ, 834, 50

Barstow, J. K., Changeat, Q., Garland, R., et al. 2020, MNRAS, 493, 4884

Barton, E. J., Yurchenko, S. N., \& Tennyson, J. 2013, MNRAS, 434, 1469

Barton, E. J., Chiu, C., Golpayegani, S., et al. 2014, MNRAS, 442, 1821

Barton, E. J., Hill, C., Czurylo, M., et al. 2017a, J. Quant. Spectr. Rad. Transf., 203,490

Barton, E. J., Hill, C., Yurchenko, S. N., et al. 2017b, J. Quant. Spectr. Rad. Transf., 187, 453

Baudino, J.-L., Mollière, P., Venot, O., et al. 2017, ApJ, 850, 150

Bauschlicher, C. W., Langhoff, S. R., \& Komornicki, A. 1990, Theor. Chim. Acta, 77, 263

Bean, J. 2013, Follow The Water: The Ultimate WFC3 Exoplanet Atmosphere Survey, HST Proposal

Bernath, P. F. 2020, J. Quant. Spectr. Rad. Transf., 240, 106687

Bittner, D. M., \& Bernath, P. F. 2018, ApJS, 235, 8

Bourgalais, J., Carrasco, N., Changeat, Q., et al. 2020, ApJ, 895, 77

Brannon, J., \& Varanasi, P. 1992, J. Quant. Spectr. Rad. Transf., 47, 237

Brooke, J. S. A., Bernath, P. F., Western, C. M., van Hemert, M. C., \& Groenenboom, G. C. 2014a, J. Chem. Phys., 141, 054310

Brooke, J. S. A., Ram, R. S., Western, C. M., et al. 2014b, ApJS, 210, 23

Brooke, J. S. A., Bernath, P. F., \& Western, C. M. 2015, J. Chem. Phys., 143, 026101

Brooke, J. S. A., Bernath, P. F., Western, C. M., et al. 2016, J. Quant. Spectr. Rad. Transf., 138, 142

Brown, L. R., Benner, D. C., Devi, V. M., Smith, M. A. H., \& Toth, R. A. 2005 J. Mol. Struct., 742, 111

Buchner, J., Georgakakis, A., Nandra, K., et al. 2014, A\&A, 564, A125

Buldyreva, J., Lavrentieva, N., \& Starikov, V. 2010a, Pressure Broadening and Shifting of Vibrotational Lines of Atmospheric Gases

Buldyreva, J., Lavrentieva, N., \& Starikov, V. 2010b, Semi-classical Calculation of Pressure-Broadened Line Widths and Pressure-Induced Line Shifts

Bunker, P. 1974, Chem. Phys. Lett., 27, 322

Burrows, A., \& Volobuyev, M. 2003, ApJ, 583, 985

Burrows, A., Marley, M. S., \& Sharp, C. M. 2000, ApJ, 531, 438

Burrows, A., Burgasser, A. J., Kirkpatrick, J. D., et al. 2002a, ApJ, 573, 394

Burrows, A., Ram, R. S., Bernath, P., Sharp, C. M., \& Milsom, J. A. 2002b, ApJ, 577, 986

Burrows, A., Dulick, M., Bauschlicher, C. W., et al. 2005, ApJ, 624, 988

Chan, A. C. H., \& Davidson, E. R. 1968, J. Chem. Phys., 49, 727

Charron, M., Anderson, T. G., \& Steinfeld, J. I. 1980, J. Chem. Phys., 73, 1494

Chen, J., Steimle, T. C., \& Merer, A. J. 2007, J. Chem. Phys., 127, 204307

Chubb, K. L., Joseph, M., Franklin, J., et al. 2018, J. Quant. Spectr. Rad. Transf., 204, 42

Chubb, K. L., Min, M., Kawashima, Y., Helling, C., \& Waldmann, I. 2020a, A\&A, 639, A3

Chubb, K. L., Tennyson, J., \& Yurchenko, S. N. 2020b, MNRAS, 493, 1531

Clark, V. H., Owens, A., Tennyson, J., \& Yurchenko, S. N. 2020, J. Quant. Spectr. Rad. Transf., 246, 106929

Cohen, J. B., \& Wilson, E. B. 1973, J. Chem. Phys., 58, 442

Coles, P. A., Yurchenko, S. N., Kovacich, R. P., Hobby, J., \& Tennyson, J. 2019a, Phys. Chem. Chem. Phys., 21, 3264

Coles, P. A., Yurchenko, S. N., \& Tennyson, J. 2019b, MNRAS, 490, 4638

Coppola, C. M., Lodi, L., \& Tennyson, J. 2011, MNRAS, 415, 487

Coursey, J., Schwab, D., Tsai, J., \& Dragoset, R. 2015, Atomic Weights and Isotopic Compositions (version 4.1) (Gaithersburg, MD: National Institute of Standards and Technology)

Darby-Lewis, D., Tennyson, J., Lawson, K. D., et al. 2018, J. Phys. B: At. Mol. Opt. Phys., 51, 185701

de Kok, R. J., Birkby, J., Brogi, M., et al. 2014, A\&A, 561, A150

Dijkerman, H., \& Ruitenberg, G. 1969, Chem. Phys. Lett., 3, 172

Drummond, B., Tremblin, P., Baraffe, I., et al. 2016, A\&A, 594, A69

Dubernet, M. L., Boudon, V., Culhane, J. L., et al. 2010, J. Quant. Spectr. Rad. Transf., 111, 2151

Dubernet, M.-L., Antony, B. K., Ba1, Y. A., , et al. 2016, J. Phys. B: At. Mol. Opt. Phys., 49, 074003

Engel, E. A., Doss, N., Harris, G. J., \& Tennyson, J. 2005, MNRAS, 357, 471

Falke, S., Tiemann, E., Lisdat, C., Schnatz, H., \& Grosche, G. 2006, Phys. Rev. A, 74, 032503

Faure, A., Wiesenfeld, L., Tennyson, J., \& Drouin, B. J. 2013, J. Quant. Spectr. Rad. Transf., 116, 79
Fernando, A. M., Bernath, P. F., Hodges, J. N., \& Masseron, T. 2018, J. Quant. Spectr. Rad. Transf., 217, 29

Feroz, F., \& Hobson, M. P. 2008, MNRAS, 384, 449

Feroz, F., Gair, J. R., Hobson, M. P., \& Porter, E. K. 2009, CQG, 26, 215003

Feroz, F., Hobson, M. P., Cameron, E., \& Pettitt, A. N. 2013, Importance Nested Sampling and the MultiNest Algorithm

Fisher, C., \& Heng, K. 2018, MNRAS, 481, 4698

Fletcher, L. N., Baines, K. H., Momary, T. W., et al. 2011, Icarus, 214, 510

Fortney, J. J., Robinson, T. D., Domagal-Goldman, S., et al. 2019, Astro2020: Decadal Survey on Astronomy and Astrophysics, science white papers, 146

Fox, K., Jennings, D. E., Stern, E. A., \& Hunnard, R. 1998, J. Quant. Spectr. Rad. Transf., 39, 473

Freedman, R. S., Marley, M. S., \& Lodders, K. 2008, ApJS, 174, 504

Freedman, R. S., Lustig-Yaeger, J., Fortney, J. J., et al. 2014, ApJS, 214, 25

Frohman, D. J., Bernath, P. F., \& Brooke, J. S. A. 2016, J. Quant. Spectr. Rad. Transf., 169, 104

Furtenbacher, T., Császár, A. G., \& Tennyson, J. 2007, J. Mol. Spectrosc., 245, 115

Gabard, T., Grigoriev, I. M., Grigorovich, N. M., \& Tonkov, M. V. 2004, J. Mol. Spectrosc., 225, 123

Gamache, R. R., \& Vispoel, B. 2018, J. Quant. Spectr. Rad. Transf., 217, 440

Gamache, R. R., Roller, C., Lopes, E., et al. 2017, J. Quant. Spectr. Rad. Transf., 203, 70

Gamache, R. R., Vispoel, B., Renaud, C. L., Cleghorn, K., \& Hartmann, L. 2019, Icarus, 326, 186

Gandhi, S., \& Madhusudhan, N. 2017, MNRAS, 472, 2334

Gandhi, S., Brogi, M., Yurchenko, S. N., et al. 2020, MNRAS, 495, 224

Gharib-Nezhad, E., \& Line, M. R. 2019, ApJ, 872, 27

Gharib-Nezhad, E., Shayesteh, A., \& Bernath, P. F. 2013, MNRAS, 432, 2043

Gharib-Nezhad, E., Heays, A. N., Bechtel, H. A., \& Lyons, J. R. 2019, J. Quant. Spectr. Rad. Transf., 239, 106649

Gordon, I. E., Rothman, L. S., Hill, C., et al. 2017, J. Quant. Spectr. Rad. Transf., 203, 3

Gorman, M., Yurchenko, S. N., \& Tennyson, J. 2019, MNRAS, 490, 1652

Grigoriev, I. M., Bouanich, J. P., Blanquet, G., Walrand, J., \& Lepere, M. 1997, J. Mol. Spectrosc., 186, 48

Grigoriev, I. M., Filippov, N. N., Tonkov, M. V., Gabard, T., \& Doucen, R. L. 2001, J. Quant. Spectr. Rad. Transf., 69, 189

Grimm, S. L., \& Heng, K. 2015, ApJ, 808, 182

Hargreaves, R. J., Gordon, I. E., Rothman, L. S., et al. 2019, J. Quant. Spectr. Rad. Transf., 232, 35

Hargreaves, R. J., Gordon, I. E., Rey, M., et al. 2020, ApJS, 247, 55

Hartmann, J.-M., Tran, H., Armante, R., et al. 2018, J. Quant. Spectr. Rad. Transf., 213, 178

Hawker, G. A., Madhusudhan, N., Cabot, S. H. C., \& Gandhi, S. 2018, ApJ, 863, L11

Hedges, C., \& Madhusudhan, N. 2016, MNRAS, 458, 1427

Hodges, J. N., \& Bernath, P. F. 2017, ApJ, 840, 81

Holka, F., \& Urban, M. 2006, Chem. Phys. Lett., 426, 252

Hou, S., \& Bernath, P. F. 2017, J. Quant. Spectr. Rad. Transf., 203, 511

Hou, S., \& Bernath, P. F. 2018, J. Quant. Spectr. Rad. Transf., 210, 44

Houdeau, J. P., Larvor, M., \& Haeusler, C. 1980, Can. J. Phys., 58, 318

Huang, X., Schwenke, D. W., Freedman, R. S., \& Lee, T. J. 2017, J. Quant. Spectr. Rad. Transf. , 203, 224

Irwin, P. G. J., Teanby, N. A., de Kok, R., et al. 2008, J. Quant. Spectr. Rad. Transf., 109, 1136

Irwin, P. G. J., Parmentier, V., Taylor, J., et al. 2020, MNRAS, 493, 106

Jacquinet-Husson, N., Armante, R., Scott, N. A., et al. 2016, J. Mol. Spectrosc., 327,31

Johnson-III, R. D. 2019, NIST Computational Chemistry Comparison and Benchmark, https://cccbdb.nist.gov

Jørgensen, U. G. 1998, SCAN Line List Database, https://www.astro.ku . $\mathrm{dk} / \sim$ uffegj/

Juncar, P., Pinard, J., Hamon, J., \& Chartier, A. 1981, Metrologia, 17, 77

Karna, S., \& Grein, F. 1992, Mol. Phys., 77, 135

Kempton, E. M.-R., Lupu, R., Owusu-Asare, A., Slough, P., \& Cale, B. 2017, PASP, 129, 044402

Kissel, A., Sumpf, B., Kronfeldt, H.-D., Tikhomirov, B., \& Ponomarev, Y. 2002, J. Mol. Spectrosc., 216, 345

Kitzmann, D., Heng, K., Oreshenko, M., et al. 2020, ApJ, 890, 174

Kleiner, I., Tarrago, G., Cottaz, C., et al. 2003, J. Quant. Spectr. Rad. Transf., 82, 293

Kramida, A., Ralchenko, Y., \& Reader, J. 2013, NIST Atomic Spectra Database - Version 5, http://www.nist.gov/pml/data/asd.cfm

Kreidberg, L., Bean, J. L., Désert, J.-M., et al. 2014, ApJ, 793, L27

Krissansen-Totton, J., Garland, R., Irwin, P., \& Catling, D. C. 2018, AJ, 156, 114

Kurucz, R., \& Bell, B. 1995, Kurucz Molecular Database, http://kurucz. harvard.edu/molecules.html 
Lacis, A. A., \& Oinas, V. 1991, J. Geophys. Res., 96, 9027

Langleben, J., Yurchenko, S. N., \& Tennyson, J. 2019, MNRAS, 488, 2332

Lee, J.-M., Fletcher, L. N., \& Irwin, P. G. J. 2012, MNRAS, 420, 170

Lee, G. K. H., Taylor, J., Grimm, S. L., et al. 2019, MNRAS, 487, 2082

Lee, G. K. H., Casewell, S. L., Chubb, K. L., et al. 2020, MNRAS, 496, 4674

Lendl, M., Cubillos, P. E., Hagelberg, J., et al. 2017, A\&A, 606, A18

Lerot, C., Blanquet, G., Bouanich, J.-P., Walrand, J., \& Lepére, M. 2006, J. Mol. Spectrosc., 238, 224

Levy, A., Lacome, N., \& Tarrago, G. 1993, J. Mol. Spectrosc., 157, 172

Li, G., Harrison, J. J., Ram, R. S., Western, C. M., \& Bernath, P. F. 2012, J. Quant. Spectr. Rad. Transf., 113, 67

Li, G., Gordon, I. E., Hajigeorgiou, P. G., Coxon, J. A., \& Rothman, L. S. 2013 , J. Quant. Spectr. Rad. Transf., 130, 284

Li, G., Gordon, I. E., Rothman, L. S., et al. 2015, ApJS, 216, 15

Li, G., Asfin, R. E., Domanskaya, A. V., \& Ebert, V. 2018, Mol. Phys., 116, 3495

Li, H. Y., Tennyson, J., \& Yurchenko, S. N. 2019, MNRAS, 486, 2351

Line, M. R., Wolf, A. S., Zhang, X., et al. 2013, ApJ, 775, 137

Lodi, L., Yurchenko, S. N., \& Tennyson, J. 2015, Mol. Phys., 113, 1559

Lyulin, O. M., \& Perevalov, V. I. 2017, J. Quant. Spectr. Rad. Transf., 201, 94

Malik, M., Grosheintz, L., Mendonça, J. M., et al. 2017, ApJ, 153, 56

Malik, M., Kitzmann, D., Mendonça, J. M., et al. 2019, AJ, 157, 170

Manne, J., Bui, T. Q., \& Webster, C. R. 2017, J. Quant. Spectr. Rad. Transf., 191, 59

Mant, B. P., Yachmenev, A., Tennyson, J., \& Yurchenko, S. N. 2018, MNRAS, 478, 3220

Mant, B. P., Chubb, K. L., Yachmenev, A., Tennyson, J., \& Yurchenko, S. N. 2019, Mol. Phys., 1

Mantz, A., Devi, V. M., Benner, D. C., et al. 2005, J. Mol. Struct., 742, 99

Masseron, T., Plez, B., Van Eck, S., et al. 2014, A\&A, 571, A47

McKemmish, L. K., Yurchenko, S. N., \& Tennyson, J. 2016, MNRAS, 463, 771

McKemmish, L. K., Masseron, T., Hoeijmakers, H. J., et al. 2019, MNRAS, 488 , 2836

McKemmish, L. K., Syme, A.-M., Borsovszky, J., et al. 2020, MNRAS, 497, 1081

Mehrotra, S. C., Mäder, H., Vreede, J. P. M. D., \& Dijkerman, H. A. 1985, Chem. Phys., 93, 115

Mikhailenko, S. N., Babikov, Y. L., \& Golovko, V. F. 2005, Atmos. Oceanic Opt., 18,685

Min, M. 2017, A\&A, 607, A9

Min, M., Hovenier, J. W., \& de Koter, A. 2005, A\&A, 432, 909

Min, M., Ormel, C. W., Chubb, K., Helling, C., \& Kawashima, Y. 2020, A\&A, 642, A28

Mizus, I. I., Alijah, A., Zobov, N. F., et al. 2017, MNRAS, 468, 1717

Mollière, P., \& Snellen, I. A. G. 2019, A\&A, 622, A139

Mollière, P., van Boekel, R., Dullemond, C., Henning, T., \& Mordasini, C. 2015, ApJ, 813, 47

Mollière, P., van Boekel, R., Bouwman, J., et al. 2017, A\&A, 600, A10

Mollière, P., Wardenier, J. P., van Boekel, R., et al. 2019, A\&A, 627, A67

Mulvihill, C. R., Alturaifi, S. A., \& Petersen, E. L. 2018, J. Quant. Spectr. Rad Transf., 217, 432

Nerf, R. B. J. 1975, J. Mol. Spectrosc., 58, 451

Olivero, J. J., \& Longbothum, R. L. 1977, J. Quant. Spectr. Rad. Transf., 17, 233

Olsen, J. 2011, Intern. J. Quantum Chem., 111, 3267

Ormel, C. W., \& Min, M. 2019, A\&A, 622, A121

Owens, A., \& Yurchenko, S. N. 2019, J. Chem. Phys., 150, 194308

Owens, A., Yurchenko, S. N., Yachmenev, A., Thiel, W., \& Tennyson, J. 2017, MNRAS, 471, 5025

Owens, A., Yachmenev, A., Tennyson, J., Thiel, W., \& Yurchenko, S. N. 2018a, MNRAS, 479, 3002

Owens, A., Yachmenev, A., Küpper, J., Yurchenko, S. N., \& Thiel, W. 2018b, Phys. Chem. Chem. Phys., 21, 3496

Owens, A., Conway, E., Tennyson, J., \& Yurchenko, S. 2020, MNRAS, 495 1927

Pascale, E., Bezawada, N., Barstow, J., et al. 2018, in Space Telescopes and Instrumentation 2018: Optical, Infrared, and Millimeter Wave, eds. M Lystrup, H. A. MacEwen, G. G. Fazio, et al., Int. Soc. Opt. Photonics (SPIE), 10698,169

Patrascu, A. T., Tennyson, J., \& Yurchenko, S. N. 2015, MNRAS, 449, 3613

Paulose, G., Barton, E. J., Yurchenko, S. N., \& Tennyson, J. 2015, MNRAS, 454, 1931

Pavlyuchko, A. I., Yurchenko, S. N., \& Tennyson, J. 2015, MNRAS, 452, 1702

Peach, G. 2017, Open Astron., 20, 516

Peach, G., \& Whittingham, I. 2009, New Astron. Rev. , 53, 227 Proceedings of the VII Serbian Conference on Spectral Line Shapes (VII SCSLSA) held in Zrenjanin, Serbia June 15th-19th 2009

Peach, G., Yurchenko, S., Chubb, K., et al. 2020, Contrib. Astron. Obs. Skalnaté Pleso, 50, 193
Petrova, T. M., Solodov, A. M., Starikov, V. I., \& Solodov, A. A. 2012, Mol. Phys., 110, 1493

Petrova, T. M., Solodov, A. M., Solodov, A. A., \& Starikov, V. I. 2013, J. Quant. Spectr. Rad. Transf., 129, 241

Petrova, T. M., Solodov, A. M., Solodov, A. A., \& Starikov, V. I. 2016, J. Mol. Spectrosc., 321, 50

Phillips, M. W., Tremblin, P., Baraffe, I., et al. 2020, A\&A, 637, A38

Pierrehumbert, R. T. 2010, Radiative Transfer in Temperature-stratified Atmospheres (Cambridge University Press), 187

Pine, A. S. 1992, J. Chem. Phys., 97, 773

Pine, A., \& Looney, J. 1987, J. Mol. Spectrosc., 122, 41

Polyansky, O. L., Kyuberis, A. A., Lodi, L., et al. 2017, MNRAS, 466, 1363

Polyansky, O. L., Kyuberis, A. A., Zobov, N. F., et al. 2018, MNRAS, 480, 2597

Prajapat, L., Jagoda, P., Lodi, L., et al. 2017, MNRAS, 472, 3648

Predoi-Cross, A., Esteki, K., Rozario, H., et al. 2016, J. Quant. Spectr. Rad. Transf., 184, 322

Ptashnik, I. V., McPheat, R., Polyansky, O. L., Shine, K. P., \& Smith, K. M. 2016, J. Quant. Spectr. Rad. Transf., 177, 92

Ram, R. S., Brooke, J. S. A., Western, C. M., \& Bernath, P. F. 2014, J. Quant. Spectr. Rad. Transf., 138, 107

Raouafi, S., Jeung, G.-H., \& Jungen, C. 2001, J. Chem. Phys., 115, 7450

Rey, M., Nikitin, A. V., Babikov, Y. L., \& Tyuterev, V. G. 2016, J. Mol. Spectrosc., 327, 138

Rey, M., Nikitin, A. V., \& Tyuterev, V. G. 2017, ApJ, 847, 105

Rivlin, T., Lodi, L., Yurchenko, S. N., Tennyson, J., \& Le Roy, R. J. 2015, MNRAS, 451, 5153

Rocchetto, M. 2017, Ph.D Thesis. University College London, London, UK

Rodgers, C. D. 2000, Inverse Methods for Atmospheric Sounding (World Scientific)

Rosmus, P., \& Meyer, W. 1977, J. Chem. Phys., 66, 13

Rothman, L. S., Gordon, I. E., Barber, R. J., et al. 2010, J. Quant. Spectr. Rad. Transf., 111, 2139

Roueff, E., Abgrall, H., Czachorowski, P., et al. 2019, A\&A, 630, A58

Salem, J., Bouanich, J.-P., Walrand, J., Aroui, H., \& Blanquet, G. 2004, J. Mol. Spectrosc., 228, 23

Salem, J., Bouanich, J.-P., Walrand, J., Aroui, H., \& Blanquet, G. 2005, J. Mol. Spectrosc., 232, 247

Schreier, F. 2017, J. Quant. Spectr. Rad. Transf., 187, 44

Sergent-Rozey, M., van Thanh, N., Rossi, I., Lacome, N., \& Levy, A. 1988, J. Mol. Spectrosc., 131, 66

Sharp, C. M., \& Burrows, A. 2007, ApJS, 168, 140

Showman, A. P., Fortney, J. J., Lian, Y., et al. 2009, ApJ, 699, 564

Sinclair, P., Duggan, P., Berman, R., Drummond, J. R., \& May, A. 1998, J. Mol. Spectrosc., 191, 258

Sing, D. K., Wakeford, H. R., Showman, A. P., et al. 2014, MNRAS, 446, 2428

Solodov, A. M., \& Starikov, V. I. 2008, Opt. Spectrosc., 105, 14

Solodov, A. M., \& Starikov, V. I. 2009, Mol. Phys., 107, 43

Sousa-Silva, C., Al-Refaie, A. F., Tennyson, J., \& Yurchenko, S. N. 2015, MNRAS, 446, 2337

Starikov, V. I., \& Protasevich, A. E. 2006, Opt. Spectrosc., 101, 523

Stevenson, K. B., Line, M. R., Bean, J. L., et al. 2017, AJ, 153, 68

Stolarczyk, N., Thibault, F., Cybulski, H., et al. 2020, J. Quant. Spectr. Rad. Transf., 240, 106676

Suenram, R., Fraser, G., Lovas, F., \& Gillies, C. 1991, J. Mol. Spectrosc., 148, 114

Takeda, Y., Kang, D.-I., Han, I., et al. 2012, PASJ, 64, 38

Tashkun, S., \& Perevalov, V. 2011, J. Quant. Spectr. Rad. Transf., 112, 1403

Tennyson, J., \& Yurchenko, S. N. 2012, MNRAS, 425, 21

Tennyson, J., \& Yurchenko, S. N. 2018, Atoms, 6, 26

Tennyson, J., Hill, C., \& Yurchenko, S. N. 2013, in 6th International Conference on Atomic and Molecular Data and their Applications ICAMDATA-2012, (New York: AIP), AIP Conf. Proc., 1545, 186

Tennyson, J., Yurchenko, S. N., Al-Refaie, A. F., et al. 2016, J. Mol. Spectrosc., 327,73

Tennyson, J., Yurchenko, S. N., Al-Refaie, A. F., et al. 2020, J. Quant. Spectr. Rad. Transf., 255, 107228

Tóbiás, R., Furtenbacher, T., Tennyson, J., \& Császár, A. G. 2019, Phys. Chem. Chem. Phys., 21, 3473

Tomasevich, G. 1970, Ph.D. Thesis, Harvard University, USA

Tsang, C. C. C., Wilson, C. F., Barstow, J. K., et al. 2010, Geophys. Lett. Res., 37

Tsiaras, A., Waldmann, I. P., Zingales, T., et al. 2018, AJ, 155, 156

Underwood, D. S., Tennyson, J., Yurchenko, S. N., et al. 2016a, MNRAS, 459, 3890

Underwood, D. S., Tennyson, J., Yurchenko, S. N., Clausen, S., \& Fateev, A. 2016b, MNRAS, 462, 4300

Upadhyay, A., Conway, E. K., Tennyson, J., \& Yurchenko, S. N. 2018, MNRAS, 477,1520 
Varanasi, P., \& Chudamani, S. 1989, J. Quant. Spectr. Rad. Transf., 41, 335 Varanasi, P., \& Chudamani, S. 1990, J. Quant. Spectr. Rad. Transf., 43, 1

Varanasi, P., \& Tejwani, G. D. T. 1972, J. Quant. Spectr. Rad. Transf., 12, 849 Voronin, B. A., Mishina, T. P., Lavrentyeva, N. N., et al. 2010, J. Quant. Spectr. Rad. Transf., 111, 2308

Voronin, B. A., Lavrentieva, N. N., Lugovskoy, A. A., et al. 2012, Atmos Oceanic Opt., 25, 27

Waldmann, I. P., Tinetti, G., Rocchetto, M., et al. 2015a, ApJ, 802, 107

Waldmann, I. P., Rocchetto, M., Tinetti, G., et al. 2015b, ApJ, 813, 13

Wang, Y., Tennyson, J., \& Yurchenko, S. N. 2020, Atoms, 8, 7

Waschull, J., Kuhnemann, F., \& Sumpf, B. 1994, J. Mol. Spectrosc., 165, 150

Wcisło, P., Gordon, I., Tran, H., et al. 2016, J. Quant. Spectr. Rad. Transf , 177, 75

XVIIIth Symposium on High Resolution Molecular Spectroscopy (HighRus2015), Tomsk, Russia

Weaver, I. C., López-Morales, M., Espinoza, N., et al. 2019, ApJ, 159, 13

Webb, R. K., Brogi, M., Gandhi, S., et al. 2020, MNRAS, 494, 108

Wende, S., Reiners, A., Seifahrt, A., \& Bernath, P. F. 2010, A\&A, 523, A58

Werner, H.-J., Knowles, P. J., Knizia, G., Manby, F. R., \& Schütz, M. 2012, WIREs Comput. Mol. Sci., 2, 242

Wilzewski, J. S., Gordon, I. E., Kochanov, R. V., Hill, C., \& Rothman, L. S. 2016,

J. Quant. Spectr. Rad. Transf., 168, 193

Woitke, P., Helling, C., Hunter, G. H., et al. 2018, A\&A, 614, A1

Wong, A., Yurchenko, S. N., Bernath, P., et al. 2017, MNRAS, 470, 882

Yadin, B., Vaness, T., Conti, P., et al. 2012, MNRAS, 425, 34
Yorke, L., Yurchenko, S. N., Lodi, L., \& Tennyson, J. 2014, MNRAS, 445, 1383

Yousefi, M., \& Bernath, P. F. 2018, ApJS, 237, 8

Yousefi, M., Bernath, P. F., Hodges, J., \& Masseron, T. 2018, J. Quant. Spectr. Rad. Transf., 217, 416

Yu, H., \& Truhlar, D. G. 2014, J. Chem. Theory Comput., 10, 2291

Yurchenko, S. N., \& Tennyson, J. 2014, MNRAS, 440, 1649

Yurchenko, S. N., Tennyson, J., Bailey, J., Hollis, M. D. J., \& Tinetti, G. 2014, Proc. Nat. Acad. Sci., 111, 9379

Yurchenko, S. N., Blissett, A., Asari, U., et al. 2016, MNRAS, 456, 4524

Yurchenko, S. N., Amundsen, D. S., Tennyson, J., \& Waldmann, I. P. 2017a, A\&A, 605, A95

Yurchenko, S. N., Tennyson, J., \& Barton, E. J. 2017b, J. Phys. Conf. Ser., 810, 012010

Yurchenko, S. N., Al-Refaie, A. F., \& Tennyson, J. 2018a, A\&A, 614, A131

Yurchenko, S. N., Williams, H., Leyland, P. C., Lodi, L., \& Tennyson, J. 2018b, MNRAS, 479, 1401

Yurchenko, S. N., Sinden, F., Lodi, L., et al. 2018c, MNRAS, 473, 5324

Yurchenko, S. N., Szabó, I., Pyatenko, E., \& Tennyson, J. 2018d, MNRAS, 480, 3397

Yurchenko, S. N., Bond, W., Gorman, M. N., et al. 2018e, MNRAS, 478, 270

Yurchenko, S. N., Mellor, T. M., Freedman, R. S., \& Tennyson, J. 2020a, MNRAS, 496, 5282

Yurchenko, S. N., Tennyson, J., Miller, S., et al. 2020b, MNRAS, 497, 2340 


\section{Appendix A: Broadening tables}

Table A.1. Molecular properties for the non-polar species with computed opacities presented in this work, where insufficient broadening parameters were found in the literature, and so those of $\mathrm{CH}_{4}$ (see Table 2) were used instead.

\begin{tabular}{lcccl}
\hline \hline Species & $\begin{array}{c}\text { DM } \\
(\mathrm{d})\end{array}$ & $\begin{array}{c}\text { MM } \\
\left(\mathrm{g} \mathrm{mol}^{-1}\right)\end{array}$ & AN & Structure Dipole Ref. \\
\hline $\mathrm{SiH}_{4}$ & 0 & 32.1 & 18 & Nonpolar \\
\hline
\end{tabular}

Notes. DM is the dipole moment, MM is the molar mass, and AN is the atomic number.

Table A.2. Molecular properties for the non-polar species with computed opacities presented in this work, where insufficient broadening parameters were found in the literature, and so those of $\mathrm{H}_{2}$ (see Table 2) were used instead.

\begin{tabular}{lccccc}
\hline \hline Species & $\begin{array}{c}\mathrm{DM} \\
(\mathrm{d})\end{array}$ & $\begin{array}{c}\mathrm{MM} \\
\left(\mathrm{g} \mathrm{mol}^{-1}\right)\end{array}$ & AN & Structure & Dipole Ref. \\
\hline $\mathrm{C}_{2}$ & 0 & 12.0 & 12 & Diatomic & Johnson-III (2019) \\
$\mathrm{H}_{3}{ }^{+}$ & 0 & 3.0 & 3 & Nonpolar & Johnson-III (2019) \\
$\mathrm{HD}^{+}$ & 0.1 & 3.0 & 2 & Diatomic & Bunker (1974) \\
\hline
\end{tabular}

Notes. DM is the dipole moment, MM is the molar mass, and AN is the atomic number.

Table A.3. Molecular properties for the non-polar species with computed opacities presented in this work, where insufficient broadening parameters were found in the literature, and so those of $\mathrm{C}_{2} \mathrm{H}_{2}$ (see Table 2) were used instead.

\begin{tabular}{lccccc}
\hline \hline Species & $\begin{array}{c}\text { DM } \\
\text { (d) }\end{array}$ & $\begin{array}{c}\text { MM } \\
\left(\mathrm{g} \mathrm{mol}^{-1}\right)\end{array}$ & AN & Structure & Dipole Ref. \\
\hline $\mathrm{C}_{2} \mathrm{H}_{4}$ & 0 & 28.0 & 16 & Nonpolar \\
$\mathrm{CH}_{3}$ & 0 & 15.0 & 9 & Nonpolar Johnson-III (2019) \\
$\mathrm{CO}_{2}$ & 0 & 44.0 & 22 & Linear & \\
$\mathrm{P}_{2} \mathrm{H}_{2}$ (trans) & 0 & 64.0 & 32 & Nonpolar \\
$\mathrm{SiO}_{2}$ & 0 & 60.1 & 30 & Linear \\
$\mathrm{SO}_{3}$ & 0 & 80.1 & 40 & Nonpolar \\
\hline
\end{tabular}

Notes. DM is the dipole moment, $\mathrm{MM}$ is the molar mass, and $\mathrm{AN}$ is the atomic number.
Table A.4. Molecular properties for the species with computed opacities presented in this work, where insufficient broadening parameters were found in the literature, and so those of $\mathrm{CO}$ (see Table 2) were used instead.

\begin{tabular}{lccccc}
\hline \hline Species & $\begin{array}{c}\text { DM } \\
\text { (d) }\end{array}$ & $\begin{array}{c}\text { MM } \\
\left(\mathrm{g} \mathrm{mol}^{-1}\right)\end{array}$ & AN & Structure & Dipole Ref. \\
\hline $\mathrm{AlCl}$ & 0.2 & 51.5 & 30 & Diatomic & Rosmus \& Meyer (1977) \\
$\mathrm{AlH}$ & 0.01 & 28.0 & 14 & Diatomic & \\
$\mathrm{AsH}_{3}$ & 0.2 & 78.0 & 36 & Non-linear & Johnson-III (2019) \\
$\mathrm{BeH}$ & 0.2 & 10.0 & 5 & Diatomic & Chan \& Davidson (1968) \\
$\mathrm{CH}$ & 0.4 & 13.0 & 7 & Diatomic & \\
$\mathrm{CN}$ & 1.5 & 26.0 & 13 & Diatomic & Johnson-III (2019) \\
$\mathrm{NO}$ & 0.2 & 30.0 & 15 & Diatomic & Johnson-III (2019) \\
$\mathrm{SiH}_{2}$ & 0.1 & 30.1 & 16 & Non-linear & \\
\hline
\end{tabular}

Notes. DM is the dipole moment, MM is the molar mass, and AN is the atomic number.

Table A.5. Molecular properties for the species with computed opacities presented in this work, where insufficient broadening parameters were found in the literature, and so those of OCS (see Table 2) were used instead.

\begin{tabular}{lccccc}
\hline \hline Species & $\begin{array}{c}\text { DM } \\
\text { (d) }\end{array}$ & $\begin{array}{c}\text { MM } \\
\left(\mathrm{g} \mathrm{mol}^{-1}\right)\end{array}$ & AN & Structure & Dipole Ref. \\
\hline $\mathrm{H}_{2} \mathrm{~S}$ & 1 & 34.1 & 18 & Polar & \\
$\mathrm{N}_{2} \mathrm{O}$ & 0.2 & 44.0 & 22 & Linear & \\
$\mathrm{NO}_{2}$ & 0.3 & 46.0 & 23 & Non-linear & Johnson-III (2019) \\
\hline
\end{tabular}

Notes. DM is the dipole moment, MM is the molar mass, and AN is the atomic number.

Table A.6. Molecular properties for the species with computed opacities presented in this work, where insufficient broadening parameters were found in the literature, and so those of $\mathrm{PH}_{3}$ (see Table 2) were used instead.

\begin{tabular}{lccccc}
\hline \hline Species & $\begin{array}{c}\text { DM } \\
(\mathrm{d})\end{array}$ & $\begin{array}{c}\mathrm{MM} \\
\left(\mathrm{g} \mathrm{mol}^{-1}\right)\end{array}$ & AN & Structure & Dipole Ref. \\
\hline $\mathrm{O}_{3}$ & 0.5 & 48.0 & 18 & Non-linear & \\
$\mathrm{PH}$ & $\sim 0.5$ & 32.0 & 16 & Diatomic & Johnson-III (2019) \\
\hline
\end{tabular}

Notes. DM is the dipole moment, MM is the molar mass, and AN is the atomic number. 
Table A.7. Molecular properties for the species with computed opacities presented in this work, where insufficient broadening parameters were found in the literature, and so those of $\mathrm{HCl}$ (see Table 2) were used instead.

\begin{tabular}{lccccc}
\hline \hline Species & $\begin{array}{c}\mathrm{DM} \\
(\mathrm{d})\end{array}$ & $\begin{array}{c}\mathrm{MM} \\
\left(\mathrm{g} \mathrm{mol}^{-1}\right)\end{array}$ & AN & Structure & Dipole Ref. \\
\hline $\mathrm{AlF}$ & 1.5 & 46.0 & 22 & Diatomic & Johnson-III (2019) \\
$\mathrm{CaF}$ & 1.2 & 59.1 & 29 & Diatomic & Raouafi et al. (2001) \\
$\mathrm{HBr}$ & 0.8 & 80.9 & 36 & Diatomic & \\
$\mathrm{HeH}$ & $\sim 1.3$ & 5.0 & 3 & Diatomic & Johnson-III (2019) \\
$\mathrm{HI}$ & 0.4 & 127.9 & 54 & Diatomic & \\
$\mathrm{MgH}$ & 1.2 & 25.3 & 13 & Diatomic & Johnson-III (2019) \\
$\mathrm{O}_{2}$ & 0 & 36.0 & 16 & Diatomic & \\
$\mathrm{PS}$ & 0.6 & 32.0 & 31 & Diatomic & Karna \& Grein (1992) \\
$\mathrm{SiH}$ & 1.2 & 29.1 & 15 & Diatomic & \\
\hline
\end{tabular}

Notes. DM is the dipole moment, MM is the molar mass, and AN is the atomic number.

Table A.8. Molecular properties for the species with computed opacities presented in this work, where insufficient broadening parameters were found in the literature, and so those of $\mathrm{NH}_{3}$ (see Table 2) were used instead.

\begin{tabular}{lccccc}
\hline \hline Species & $\begin{array}{c}\text { DM } \\
\text { (d) }\end{array}$ & $\begin{array}{c}\text { MM } \\
\left(\mathrm{g} \mathrm{mol}^{-1}\right)\end{array}$ & AN & Structure & Dipole Ref. \\
\hline $\mathrm{H}_{3} \mathrm{O}^{+}$ & $\sim 1.5$ & 19.0 & 11 & Non-linear & Johnson-III (2019) \\
$\mathrm{P}_{2} \mathrm{H}_{2}$ (cis) & 1.4 & 64.0 & 32 & Non-linear & \\
\hline
\end{tabular}

Notes. DM is the dipole moment, MM is the molar mass, and AN is the atomic number.

Table A.9. Molecular properties for the species with computed opacities presented in this work, where insufficient broadening parameters were found in the literature, and so those of HF (see Table 2) were used instead.

\begin{tabular}{|c|c|c|c|c|c|}
\hline Species & $\begin{array}{l}\text { DM } \\
\text { (d) }\end{array}$ & $\begin{array}{c}\mathrm{MM} \\
\left(\mathrm{g} \mathrm{mol}^{-1}\right)\end{array}$ & $\mathrm{AN}$ & Structure & Dipole Ref \\
\hline $\mathrm{CP}$ & 2.1 & 43.0 & 21 & Diatomic & \\
\hline $\mathrm{CS}$ & 2.0 & 44.1 & 22 & Diatomic & Johnson-III (2019) \\
\hline $\mathrm{FeH}$ & 2.0 & 56.9 & 27 & Diatomic & Chen et al. (2007) \\
\hline $\mathrm{LiH}^{+}$ & $\sim 2$ & 8.0 & 4 & Diatomic & Johnson-III (2019) \\
\hline $\mathrm{MgF}$ & 1.8 & 43.3 & 21 & Diatomic & \\
\hline $\mathrm{NH}$ & 0.5 & 15.0 & 8 & Diatomic & Johnson-III (2019) \\
\hline NS & 1.8 & 46.1 & 23 & Diatomic & Johnson-III (2019) \\
\hline $\mathrm{OH}$ & 1.7 & 17.0 & 9 & Diatomic & Johnson-III (2019) \\
\hline $\mathrm{OH}^{+}$ & $\sim 2$ & 17.0 & 9 & Diatomic & Johnson-III (2019) \\
\hline $\mathrm{PO}$ & 1.9 & 47.0 & 23 & Diatomic & Johnson-III (2019) \\
\hline $\mathrm{ScH}$ & 1.7 & 46.0 & 22 & Diatomic & Johnson-III (2019) \\
\hline $\mathrm{SiS}$ & 1.7 & 60.2 & 30 & Diatomic & Johnson-III (2019) \\
\hline $\mathrm{TiH}$ & $\sim 2$ & 48.9 & 23 & Diatomic & Johnson-III (2019) \\
\hline
\end{tabular}

Notes. DM is the dipole moment, MM is the molar mass, and AN is the atomic number.
Table A.10. Molecular properties for the species with computed opacities presented in this work, where insufficient broadening parameters were found in the literature, and so those of $\mathrm{H}_{2} \mathrm{CO}$ (see Table 2) were used instead.

\begin{tabular}{lccclc}
\hline \hline Species & $\begin{array}{c}\text { DM } \\
\text { (d) }\end{array}$ & $\begin{array}{c}\text { MM } \\
\left(\mathrm{g} \mathrm{mol}^{-1}\right)\end{array}$ & AN & Structure & Dipole Ref. \\
\hline $\mathrm{CH}_{3} \mathrm{Cl}$ & 1.9 & 50.5 & 26 & Non-linear & \\
$\mathrm{CH}_{3} \mathrm{~F}$ & 1.9 & 34.0 & 18 & Non-linear & Johnson-III (2019) \\
$\mathrm{H}_{2} \mathrm{O}_{2}$ & 2.3 & 34.0 & 18 & Non-linear & \\
$\mathrm{HNO}_{3}$ & 2.2 & 63.0 & 32 & Non-linear & Johnson-III (2019) \\
\hline
\end{tabular}

Notes. DM is the dipole moment, MM is the molar mass, and AN is the atomic number.

Table A.11. Molecular properties for the species with computed opacities presented in this work, where insufficient broadening parameters were found in the literature, and so those of HCN (see Table 2) were used instead.

\begin{tabular}{lccccc}
\hline \hline Species & $\begin{array}{c}\text { DM } \\
(\mathrm{d})\end{array}$ & $\begin{array}{c}\mathrm{MM} \\
\left(\mathrm{g} \mathrm{mol}^{-1}\right)\end{array}$ & AN & Structure & Dipole Ref \\
\hline $\mathrm{AlO}$ & 4.2 & 43.0 & 21 & Diatomic & Johnson-III (2019) \\
$\mathrm{CaH}$ & 2.9 & 41.1 & 21 & Diatomic & $\begin{array}{c}\text { Holka \& Urban (2006) } \\
\mathrm{CaO}\end{array}$ \\
8.7 & 56.1 & 28 & Diatomic & Yu \& Truhlar (2014) \\
$\mathrm{CrH}$ & 3.9 & 53.0 & 25 & Diatomic & Johnson-III (2019) \\
$\mathrm{KCl}$ & 10.2 & 74.6 & 36 & Diatomic & Johnson-III (2019) \\
$\mathrm{KF}$ & 8.6 & 58.1 & 28 & Diatomic & Johnson-III (2019) \\
$\mathrm{LiCl}$ & 7.1 & 42.4 & 20 & Diatomic & Johnson-III (2019) \\
$\mathrm{LiF}$ & 6.3 & 25.9 & 12 & Diatomic & Johnson-III (2019) \\
$\mathrm{LiH}$ & 5.9 & 8.0 & 4 & Diatomic & Johnson-III (2019) \\
$\mathrm{MgO}$ & 6.2 & 40.3 & 20 & Diatomic & Johnson-III (2019) \\
$\mathrm{NaCl}$ & 9.0 & 58.4 & 28 & Diatomic & Johnson-III (2019) \\
$\mathrm{NaF}$ & 8.1 & 42.0 & 20 & Diatomic & Johnson-III (2019) \\
$\mathrm{NaH}$ & 6.0 & 24.0 & 12 & Diatomic & Johnson-III (2019) \\
$\mathrm{PN}$ & 2.8 & 45.0 & 22 & Diatomic & Johnson-III (2019) \\
$\mathrm{SH}$ & 2.7 & 33.1 & 17 & Diatomic & \\
$\mathrm{SiO}$ & 3.1 & 44.1 & 22 & Diatomic & Badreddine et al. (2013) \\
$\mathrm{TiO}$ & $\sim 3$ & 63.9 & 30 & Diatomic & Bauschlicher et al. (1990) \\
$\mathrm{VO}$ & 3.4 & 66.9 & 31 & Diatomic & Suenram et al. (1991) \\
\hline
\end{tabular}

Notes. DM is the dipole moment, MM is the molar mass, and AN is the atomic number. 